\title{
Inhibiting Wee1 and ATR kinases produces tumor- selective synthetic lethality and suppresses metastasis
}

\author{
Amirali B. Bukhari, Cody W. Lewis, Joanna J. Pearce, Deandra Luong, Gordon K. Chan, and Armin M. Gamper \\ Cross Cancer Institute, Department of Oncology, University of Alberta, Edmonton, Canada.
}

\begin{abstract}
We used the cancer-intrinsic property of oncogene-induced DNA damage as the base for a conditional synthetic lethality approach. To target mechanisms important for cancer cell adaptation to genotoxic stress and thereby to achieve cancer cellspecific killing, we combined inhibition of the kinases ATR and Wee1. Wee1 regulates cell cycle progression, whereas ATR is an apical kinase in the DNA-damage response. In an orthotopic breast cancer model, tumor-selective synthetic lethality of the combination of bioavailable ATR and Wee1 inhibitors led to tumor remission and inhibited metastasis with minimal side effects. ATR and Wee1 inhibition had a higher synergistic effect in cancer stem cells than in bulk cancer cells, compensating for the lower sensitivity of cancer stem cells to the individual drugs. Mechanistically, the combination treatment caused cells with unrepaired or under-replicated DNA to enter mitosis leading to mitotic catastrophe. As these inhibitors of ATR and Wee1 are already in phase I/II clinical trials, this knowledge could soon be translated into the clinic, especially as we showed that the combination treatment targets a wide range of tumor cells. Particularly, the antimetastatic effect of combined Wee1/ATR inhibition and the low toxicity of ATR inhibitors compared with Chk1 inhibitors have great clinical potential.
\end{abstract}

\section{Introduction}

The DNA-damage response (DDR) senses DNA damage and replication stress and orchestrates the cellular response to protect the cell and organism from genotoxic insults. These signal transduction pathways include the choreography of DNA repair, cell cycle control, and cell fate decision, among others (1). Owing to their dysregulated proliferation, the genomic integrity of cancer cells is particularly threatened by DNA damage and replication stress, but also by metabolic, mitotic, oxidative, and proteotoxic stresses (2). Furthermore, during tumorigenesis cells often lose DDR mechanisms, leading to increased genomic instability (3). These DNA repair/DNA damage signaling defects and/or the increased genotoxic stress make cancer cells heavily dependent on the (remaining) intact DDR pathways.

Synthetic lethality refers to an interaction between two genes when the perturbation of either gene alone is viable but the simultaneous perturbation of both genes leads to cell death. The discovery that breast cancer cells with mutations in homologous recombination proteins BRCA1 or BRCA2 are hypersensitive to poly(ADP-ribose) polymerase (PARP) inhibitors $(4,5)$ led to therapeutic approaches targeting cancer cells with deficiencies in one DDR pathway by inhibition of an alternative DDR pathway. However, as this approach only targets cells with a defective DDR, it is bound to only affect a subset of cancers or populations within a tumor. Resistance can arise by reactivation of the defective pathway. Conditional synthetic lethality refers to synthetic lethality observed only under certain circumstances, such as genetic

Conflict of interest: The authors have declared that no conflict of interest exists. License: Copyright 2019, American Society for Clinical Investigation.

Submitted: June 1, 2018; Accepted: January 8, 2019.

Reference information: J Clin Invest. 2019;129(3):1329-1344.

https://doi.org/10.1172/JCl122622. background or metabolic state of cells or cellular environment (6). In view of the inter- and intratumor heterogeneity commonly observed, to achieve the necessary therapeutic window for a wide range of tumors it is necessary to identify a common condition upon which to base cancer-selective conditional synthetic lethality. Oncogene-induced DNA damage is a common feature of cancer cells leading to high levels of replication stress as well as mitotic stress in cancer cells compared with normally proliferating cells $(7,8)$. Based on this tumor-specific property, we tested whether increasing genotoxic stress and simultaneously inhibiting an important rescue pathway would lead to cancer cell-selective death by evaluating the efficacy of combined ATR and Wee1 inhibition on cancer cell eradication. Wee1 is a kinase controlling $\mathrm{G} / \mathrm{M}$ and S phase checkpoints via phosphorylation of the cyclindependent kinases CDK1 and CDK2. Furthermore, Wee1 inhibition prolongs mitosis in a range of cancer cells and makes them more susceptible to chemotherapy-induced mitotic catastrophe (9). Ataxia telangiectasia and Rad3 related (ATR) is the apical kinase of a DDR pathway. ATR is considered the main mediator in the DDR to replication stress (7), including signaling to cell cycle checkpoints via Chk1 and regulating repair by promoting extensive DNA end resection needed for homologous recombination (10-12). Bioavailable selective ATR inhibitors (AZD6738 by AstraZeneca; VX-970 and VX-803 by Merck) as well as the Wee1 inhibitor AZD1775 have recently entered phase I/II clinical trials in combination with radiation or chemotherapeutics.

Here, we report that the tumor-selective synthetic lethality of the combination of ATR and Wee1 inhibitors leads to tumor shrinkage and suppresses metastasis. Using an orthotopic breast cancer xenograft mouse model we show that combination treatment leads to complete remission in 6 of 9 cases, inhibits tumor spread, and prolongs survival. Our toxicity studies show that the combination treatment is associated with minimal side effects. 
Table 1. Synergistic cancer cell killing by ATR and Wee1 inhibition

$\begin{array}{lccccc}\text { Cell Line } & \text { AZD6738 (ATRi) } & \text { AZD1775 (Wee1i) } & \text { Bliss Cl } & \text { ATRi IC }_{50} & \text { Wee1i IC }_{50} \\ \text { MDA-MB-231 } & 300 \mathrm{nM} & 100 \mathrm{nM} & 0.60 & 540 \mathrm{nM} & 190 \mathrm{nM} \\ \text { MCF7 } & 300 \mathrm{nM} & 100 \mathrm{nM} & 0.65 & 840 \mathrm{nM} & 280 \mathrm{nM} \\ \text { Zr-75-1 } & 500 \mathrm{nM} & 300 \mathrm{nM} & 0.74 & 1,120 \mathrm{nM} & 1,270 \mathrm{nM} \\ \text { T-47D } & 750 \mathrm{nM} & 500 \mathrm{nM} & 0.83 & 1,120 \mathrm{nM} & 340 \mathrm{nM} \\ \text { MDA-MB-468 } & 300 \mathrm{nM} & 100 \mathrm{nM} & 0.56 & 2,580 \mathrm{nM} & 520 \mathrm{nM} \\ \text { MDA-MB-175-VII } & 300 \mathrm{nM} & 100 \mathrm{nM} & 0.34 & 3,740 \mathrm{nM} & 460 \mathrm{nM} \\ \text { Sk-Br-3 } & 300 \mathrm{nM} & 100 \mathrm{nM} & 0.57 & 5,165 \mathrm{nM} & 570 \mathrm{nM} \\ \text { U-2 0S } & 300 \mathrm{nM} & 100 \mathrm{nM} & 0.54 & 550 \mathrm{nM} & 160 \mathrm{nM} \\ \text { hTERT-HME1 } & 300 \mathrm{nM} & 100 \mathrm{nM} & 1.07 & 2,400 \mathrm{nM} & 660 \mathrm{nM} \\ & 750 \mathrm{nM} & 500 \mathrm{nM} & 1.11 & 2,400 \mathrm{nM} & 660 \mathrm{nM} \\ \text { MCF10A } & 300 \mathrm{nM} & 100 \mathrm{nM} & 1.09 & 3,790 \mathrm{nM} & 615 \mathrm{nM} \\ & 750 \mathrm{nM} & 500 \mathrm{nM} & 1.08 & 3,790 \mathrm{nM} & 615 \mathrm{nM}\end{array}$

$\mathrm{IC}_{50}$ values and Bliss combination indices (Cls) at indicated drug concentrations were calculated from at least 3 independent experiments. A Bliss $\mathrm{Cl}$ of less than 1 indicates synergy, a $\mathrm{Cl}$ of less than 0.7 strong synergy, and a $\mathrm{Cl}$ of greater than 1 antagonism. double-strand breaks. ATR activation is important for S and G2/M checkpoint signaling and DNA-damage repair by homologous recombination (10).

To test whether Wee1 inhibition activates ATR, we incubated cancer cells for 2 hours with the Wee1 inhibitor AZD1775. Immunoblots of cell lysates show that AZD1775 treatment leads to phosphorylation of Chk1 serine 345, a target site of ATR (Figure 1A). ATR activation was confirmed by cotreatment with 2 ATRselective inhibitors, AZD6738 andETP-46464, which suppressed AZD1775-induced Chk1 phosphorylation (Figure 1A, lanes 1-4), and is observed in breast cancer (MDA-MB-231) and osteosarcoma (U-2 OS) cells, indicating that it is unlikely cancer type specific (Figure $1 \mathrm{~A}$ and Supplemental Figure 1; supplemental material available online with this article; https://doi.org/10.1172/JCI122622DS1). ATR Rapidly proliferating tissues, such as the ileum or bone marrow, showed no signs of renewal defects. Synergistic cell killing by inhibition of ATR and Wee1 was observed in cancer cells from various tissue origins, but not in untransformed cells. Mechanistic studies using pulses of reversible inhibition during the cell cycle showed that combined ATR and Wee1 inhibition during S and G2/M phase cooperates to kill cancer cells. Furthermore, live cell imaging studies revealed that combined ATR/Wee1 inhibition causes cells to enter mitosis with unrepaired/under-replicated DNA leading to mitotic catastrophe. As the studied ATR and Wee1 inhibitors are already in phase I/II clinical trials, this knowledge could soon be translated into the clinic.

\section{Results}

Synergistic cell killing of cancer cells by ATR and Wee1 inhibition in vitro. CDK1/2 activity is regulated by inhibitory phosphorylation at tyrosine 15 by the protein kinase Wee1 that is counteracted by the phosphatase Cdc25. CDK1 activity regulates entry into and exit out of mitosis (13-17), and we recently showed that Wee1 inhibition in breast cancer cells promotes premature mitosis, prolongs mitosis, and promotes paclitaxel-induced mitotic catastrophe (9). In addition to regulating entry into mitosis, screens identified an important role for Wee1 in the maintenance of genomic integrity during DNA replication. Wee1 knockdown or inhibition leads to upregulation of phosphorylated $\mathrm{H} 2 \mathrm{AX}(\gamma \mathrm{H} 2 \mathrm{AX})$, a readout for DNA damage, in $S$-phase cells (18-20). The underlying mechanisms remain poorly understood and seemingly conflicting data led to 2 models proposing either that Wee1 controls genomic stability during replication by regulating origin firing (19), or that it regulates the processing of stalled replication forks by the Mus81-Eme1 endonuclease (20).

The protein kinase ATR is constitutively bound by ATRinteracting protein (ATRIP) and is activated by replication protein A-coated (RPA-coated) single-stranded DNA, structures that can arise from stalled replication forks or resected DNA doublestrand breaks (1). Unsurprisingly, ATR plays a crucial role in the response to replication stress - likely the reason for it being an essential gene $(21,22)$ - and to ionizing radiation-induced DNA activation by AZD1775 is potentiated by DNA-damaging agents, such as ionizing radiation (Supplemental Figure 1). The activation of ATR by Wee1 inhibition prompted us to study the combinatorial effect of Wee1 and ATR inhibition on cancer cell killing. Five thousand cells were plated and incubated with different concentrations of AZD1775 and AZD6738 for 4 days before measuring surviving cells by crystal violet staining and colorimetry (23). We observed synergistic cell killing by ATR and Wee1 inhibition in all tested cancer cell lines (Table 1 and Supplemental Figure 2C), including the human breast cancer cell lines MDA-MB-231, MCF7, and Zr-75-1 (Figure 1, B-D), but not in nontumorigenic MCF10A and immortalized mammary epithelial cells (hTERT-HME1) (Figure 1, E and F), as demonstrated in Loewe plots and calculated Bliss combination indices (CIs) (24). A CI below 1 indicates synergy. The synergistic cell killing we observe with Wee1 and ATR inhibitors is unlikely due to off-target effects, because several ATR inhibitors (including ETP-46464 and VE821, Supplemental Figure 3) and knockdown of Wee1 with siRNA (Supplemental Figure 2D) show cooperative lethality as well. Importantly, and in agreement with a conditional synthetic lethality of Wee1 and ATR based on DNA damage, a favorable therapeutic window for the combination treatment is provided by the increased oncogenic stress in cancer cells, as no cooperative lethality is observed in MCF10A and hTERT-HME1. This is in stark contrast to inhibition of the ATR downstream target Chk1. MCF10A alone and to combined Wee1 and Chk1 inhibition (Supplemental Figure 4). Depletion or inhibition of Chk1, but not of ATR, has previously been shown to cause DNA damage in normal cells (25), likely explaining the toxicity of Chk1 inhibitors observed in the clinic $(26,27)$. Several studies have shown that in the absence of exogenous genotoxic stress ATR inhibitors are well tolerated $(28,29)$ and cells from Seckel syndrome patients, who have hypomorphic levels of ATR, do not show increased DNA damage levels (30), indicating that low ATR activity is sufficient to respond to the endogenous genotoxic stress in normal cells.

Combination treatment of cancer cells with ATR and Wee1 inhibitors leads to centromere fragmentation and mitotic catastrophe. and hTERT-HME1 are very sensitive to the Chk1 inhibitor UCN-01 

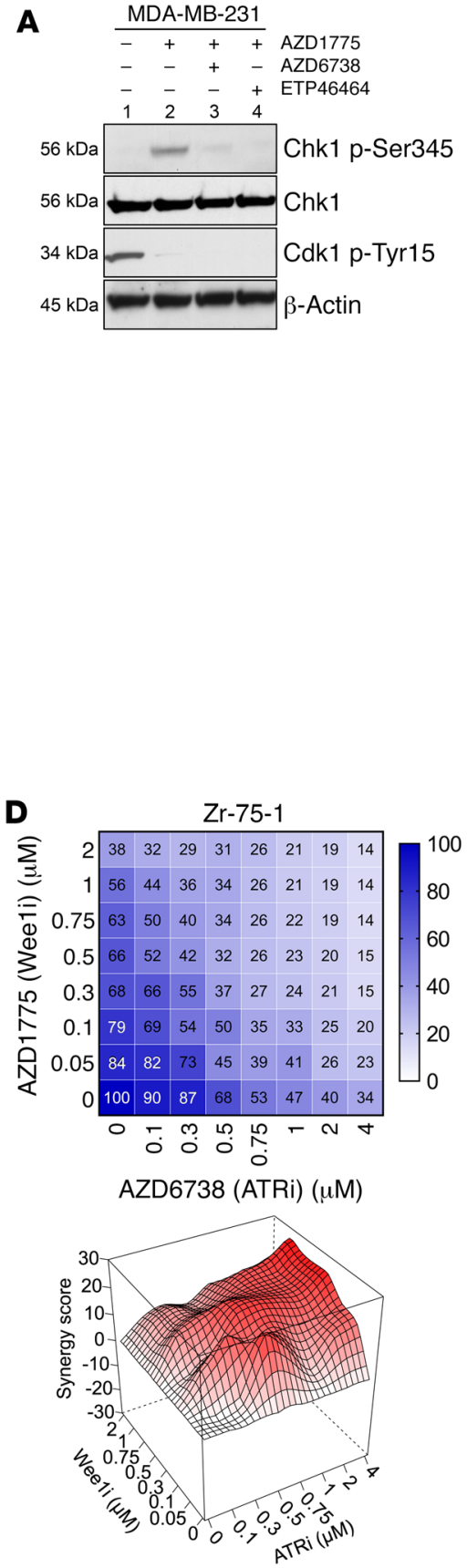
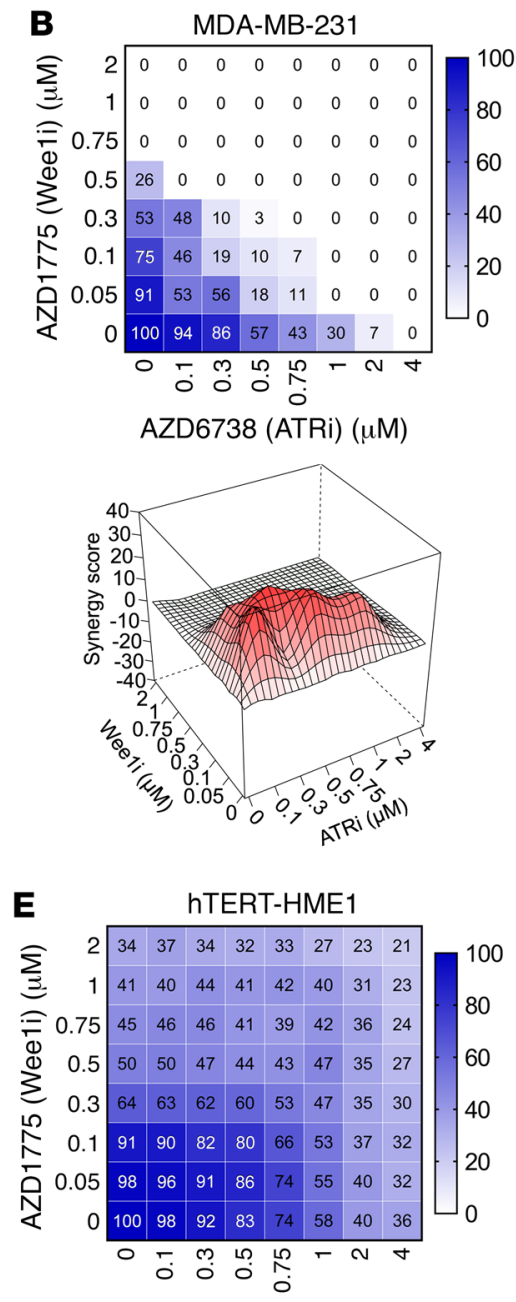

AZD6738 (ATRi) $(\mu \mathrm{M})$

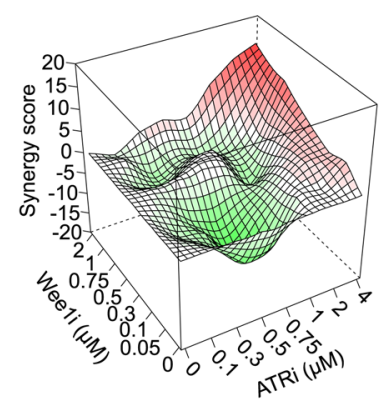

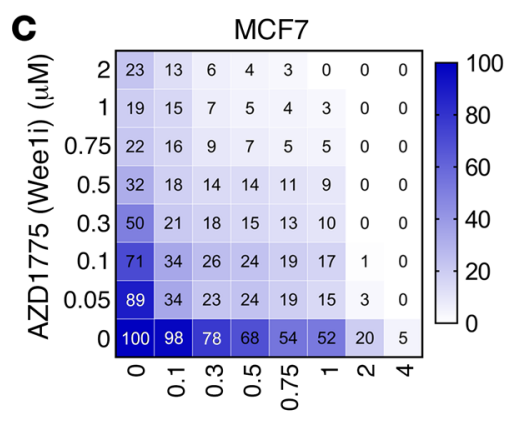

AZD6738 (ATRi) $(\mu \mathrm{M})$
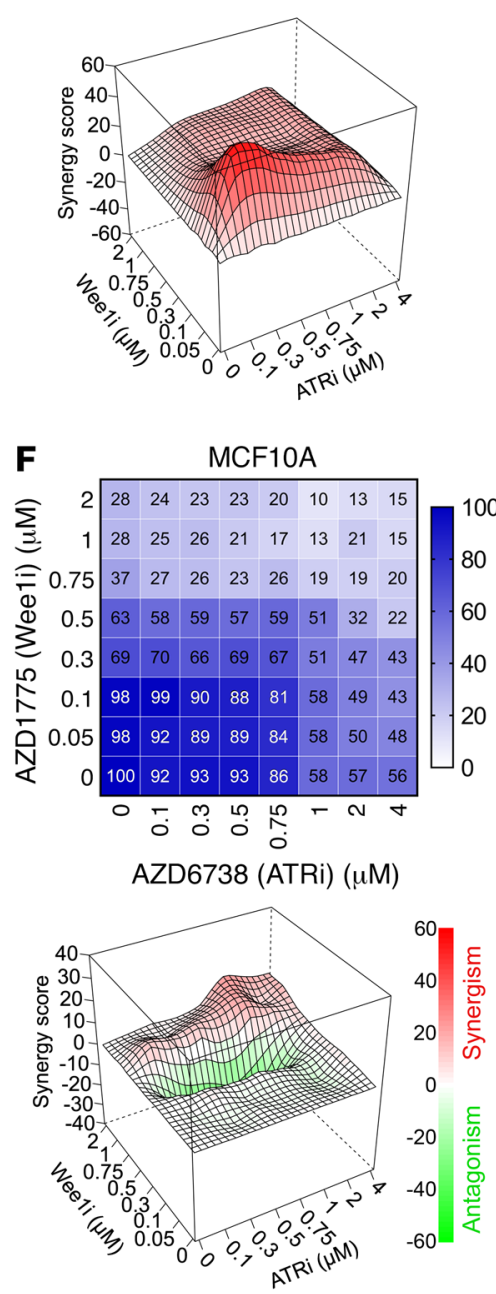

Figure 1. Wee1 inhibition activates ATR and shows synergistic cancer cell killing with ATR inhibition. (A) MDA-MB-231 cells were incubated with the indicated inhibitors for Wee1 (AZD1775) or ATR (AZD6738, ETP46464). After 2 hours, cell lysates were harvested and probed for Chk1 and CDK1 phosphorylation by immunoblotting. (B-F) MDA-MB-231, MCF7, Zr-75-1, hTERT-HME1, or MCF10A cells were treated for 4 days with a combination of up to $4 \mu \mathrm{M}$ AZD6738 and up to $2 \mu \mathrm{M}$ AZD1775. Survival was assayed by crystal violet staining and each experiment was repeated at least 4 times. Color bars indicate percentage survival normalized to untreated cells. Representative cooperativity screens and Loewe plots for drug cooperativity are shown.

Several reproductive cell death modes can lead to the inability of a cell to reproduce after exposure to genotoxic stress $(31,32)$. While treated cells with intact cell cycle checkpoint function tend to senesce, the major death mechanism after exposure to DNAdamaging agents for cells with defects in cell cycle checkpoints and impaired DNA repair mechanisms is mitotic catastrophe. Mitotic catastrophe occurs when cells enter mitosis prematurely before the completion of DNA repair and/or DNA replication, resulting in dysregulated or failed mitosis, and can lead to delayed apoptosis, senescence, or even necrosis.

We used live cell microscopy to address whether cell death by Wee1 and/or ATR inhibition requires cells to enter mitosis. Cancer cell lines display variable intraline (within their population) response to drug treatments (33). Therefore, monitoring individual cell fates with time-lapse microscopy is essential to understanding the cell cycle response of cancer cells to drug treatment. Breast 
cancer cell lines stably expressing GFP-tubulin and mCherryhistone $\mathrm{H} 2 \mathrm{~B}$ enabled us to track the fates of individual cells and their progenies. Our data for MDA-MB-231 show that, unlike Wee1 inhibition ( $P=0.0387$, one-way ANOVA) (9), ATR inhibition alone does not prolong mitosis (Figure 2, A and B). However, when ATR and Wee1 inhibition are combined, mitosis is significantly longer $(P<0.0001$, one-way ANOVA) (Figure $2, \mathrm{~A}$ and $\mathrm{B}$ ) and commonly leads to cell death (Figure 2, C and D). The median time between nuclear envelope breakdown and anaphase in control cells or cells treated with AZD6738, AZD1775, or the combination is 35, 45,160 , or 325 minutes, respectively (Figure 2B). Cell death is observed during failed mitosis, after mitotic slippage (when cells have aborted mitosis, as evidenced by the disappearance of the mitotic spindle without cytokinesis), or in interphase after cytokinesis (often with visible micronucleation) (Figure 2, C and D, and Supplemental Figure 5A). Mitotic duration seems to correlate with cell death observed during mitosis, with $0,3.6 \%, 28.6 \%$, or $64.3 \%$ of MDA-MB-231 cells dying in mitosis when treated with vehicle, AZD6738, AZD1775, or combined AZD6738/AZD1775, respectively (Figure 2D). While ATR inhibition kills $44.6 \%$ of the cells, most of the cell death occurs during interphase in daughter cells. We do not observe interphase death in cells before aborted or completed mitosis. This clearly indicates the importance of cells entering mitosis, presumably with unrepaired or underreplicated DNA, for cell death and shows that mitotic defects can lead to delayed cell death in daughter cells.

Mitotic cells with under-replicated genomes (MUGs) were discovered 30 years ago (34). Mitotic defects observed in these cells commonly include centromere fragmentation (35), characterized by the formation of centromere clusters spatially separated from the main mass of chromosomes. As the majority of cells treated with combined ATR and Wee1 inhibitors died in mitosis, we synchronized cells in S phase by a double thymidine block and inhibited ATR and/or Wee1 after release. Four hours after G1/S release, cells were fixed and stained for tubulin, centromeres, and DNA (Figure 2E). Wee1 inhibition, but particularly combined ATR/Wee1 inhibition, leads to an increase in mitotic cells (Figure $2 \mathrm{~F}$ ) in the breast cancer cell lines MDA-MB-231 and T-47D, as well as in HeLa cells (Supplemental Figure 5B). Furthermore, the majority of the mitotic cells in the combination treatment group show centromere fragmentation, as seen by the clustering of centromeres and kinetochores and their separation form the bulk condensed chromatin (compare mitotic cells treated with combined AZD6738 and AZD1775 to DMSO control in Figure 2E and Supplemental Figure 5B).

Events in $S$ phase and $G 2 / M$ phase contribute to the synergistic cancer cell killing by the combination treatment of cancer cells with ATR and Wee1 inhibitors. To estimate the contribution of abrogation of cell cycle checkpoints and DNA-damage repair to overall cell killing, we evaluated the impact of ATR and/or Wee1 activity during phases of the cell cycle on cancer cell survival. As this requires the ability to switch ATR and Wee1 activity on and off, we tested the reversible nature of inhibition by AZD6738 and AZD1775. Mock- or AZD6738-treated cells were UV-irradiated and ATR activation measured by Chk1 phosphorylation (Figure 3A). AZD6738 washout leads to ATR reactivation within 1 hour, as evidenced by restoration of high Chk1 phospho-S345 levels.
AZD1775 treatment of cells reduces CDK1 phospho-Y15 levels, confirming that Wee 1 is the primary kinase phosphorylating CDK1 at tyrosine 15 (Figure 3B). Washout of AZD1775 restores Wee1 kinase activity to full levels in less than 2 hours, as shown by the reestablishment of normal CDK1 phospho-Y15 levels. Having established that ATR and Wee1 inhibition can be reversed within approximately 1 hour, we next synchronized U-2 OS cells by a thymidine-nocodazole block (Figure $3 \mathrm{C}$ ) as described previously (10). At various times after nocodazole release and for different durations, cells were pulse-treated with $1 \mu \mathrm{M}$ AZD6739 and/or $300 \mathrm{nM}$ AZD1775 by addition and subsequent washout as indicated: from +10 to +16 hours (roughly late G1 to G2), from +18 to +22 hours (late G2 into mitosis), from +10 to +22 hours, for a full cell cycle starting from late G1, or for the entire period of 4 days. All cells were assayed for survival after 4 days by measuring crystal violet staining compared with mock-treated cells. As discussed previously, treatment for the entire time window with a combination of AZD6738 and AZD1775 leads to strong synergy (Figure 3D). Inhibition of ATR or Wee1 alone for short intervals, during S phase or late G2/mitosis (Figure 3D), had no significant effect on survival, indicating that cells were able to recover from transient ATR or Wee1 inhibition for the indicated time intervals. On the other hand, prolonged inhibition, from late G1 into mitosis, leads to significant cell killing by the single agents $(P<0.0001$, one-way ANOVA), comparable to inhibition for an entire cell cycle. Interestingly, combined ATR and Wee1 inhibition for just the short periods encompassing S phase $(+10$ to +16 hours) or from late $\mathrm{G} 2$ into mitosis ( +18 to +22 hours) leads to killing of approximately half of the cells $(P<0.0001$, one-way ANOVA). Yet when ATR and Wee1 are both inhibited from late G1 into mitosis (+10 to +22 hours), less than $10 \%$ of the cells survive, indicating not only a strong synergy between the 2 inhibitors, but also the contribution of events during both cell cycle intervals (G1 to G2; G2 and mitosis) the inhibitors were active (Figure 3D). Combination treatment for an entire normal cell cycle interval further increased cell killing to levels comparable to treatment for the entire 4 days.

We also tested inhibitor-induced changes in cell cycle profiles in cells synchronized by a thymidine-nocodazole block, if AZD6738 and/or AZD1775 were added to G1 cells 6 hours after release. Flow cytometric analysis of propidium iodide-stained cells shows a significant increase of cells with DNA content between $2 \mathrm{n}$ and $4 \mathrm{n}$ at 14 hours after nocodazole release in the combined ATR- and Wee1-inhibitor-treated group compared with control (Figure 3C). The DNA content indicates delayed S phase or entry into G2/mitosis with under-replicated genomes. The latter is more likely, because many cells retain a DNA content below $4 \mathrm{n}$ even several hours later. Combined with our observation that cells treated with both ATR and Wee1 inhibitors show frequent centromere fragmentation in mitosis (Figure 2, E and F), a hallmark of under-replicated cells entering mitosis, the inhibitor-induced shift in DNA-content profile underlines the synergistic contribution of reversible ATR/Wee1 inhibition during S and G2/M phases in causing mitotic catastrophe.

Combined inhibition of ATR and Wee1 leads to increased DNA damage in tumors in vivo. AZD1775 and AZD6738 are both bioavailable and can be administered to mice by oral gavage. To test synthetic lethality of the combination of Wee1 and ATR inhibitors 
A

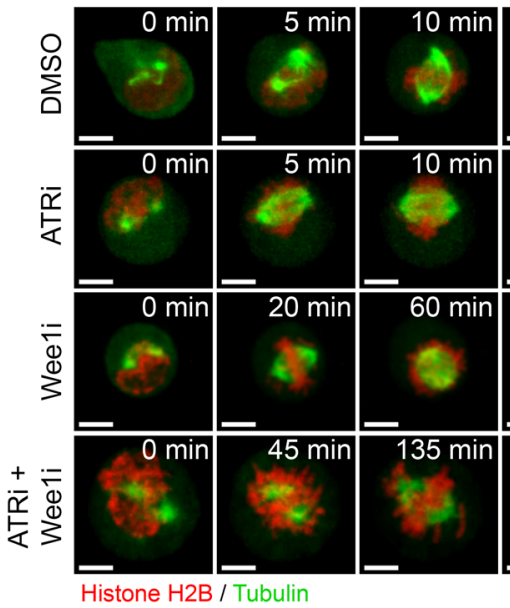

C

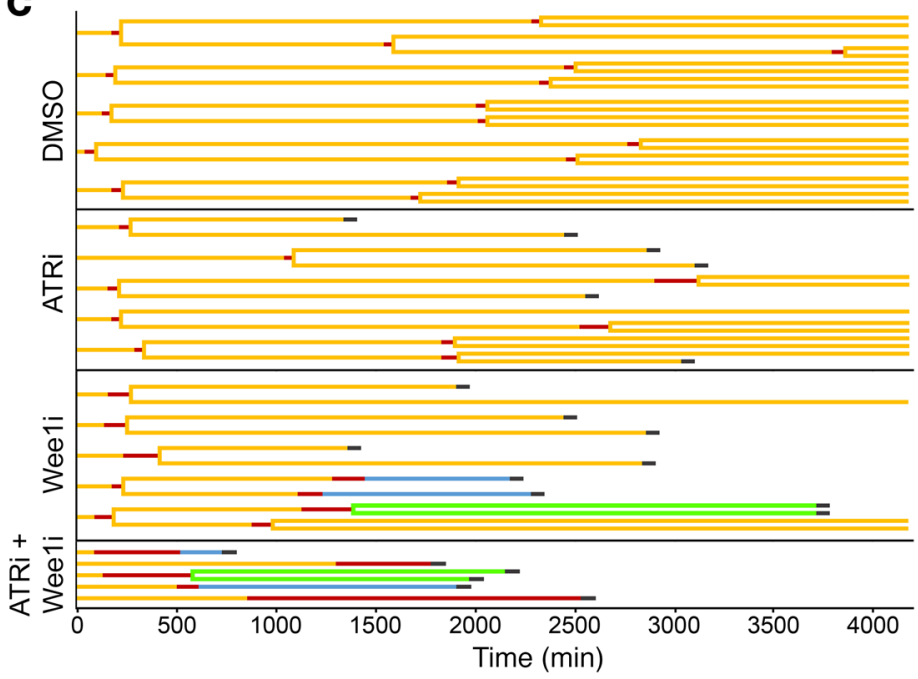

MDA-MB-231
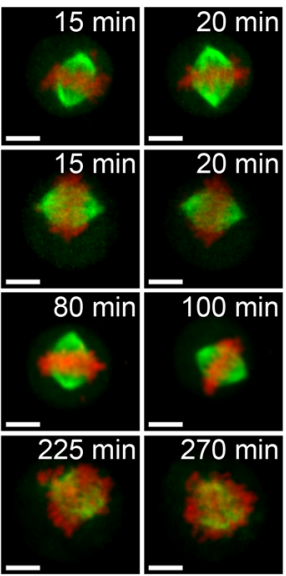

B

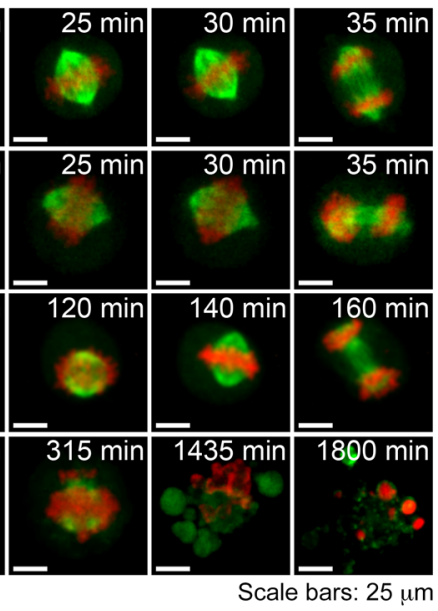

Scale bars: $25 \mu \mathrm{m}$

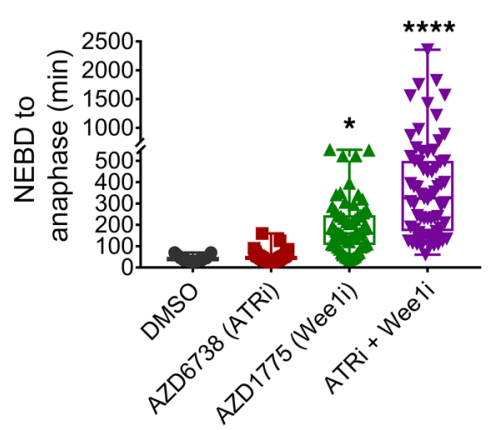

E

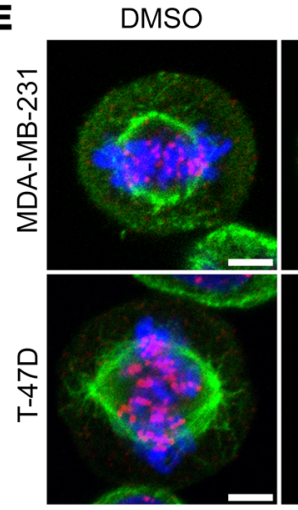

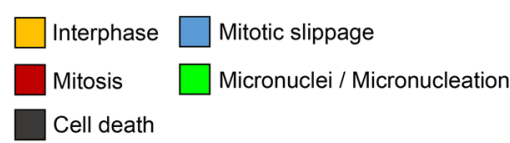

D

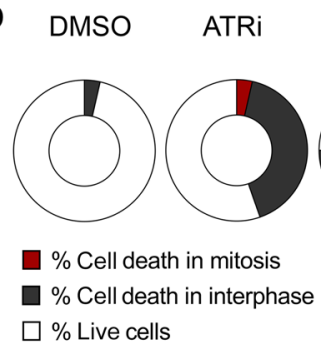

terphase

$\square \%$ Live cells
ATRi + Wee1i

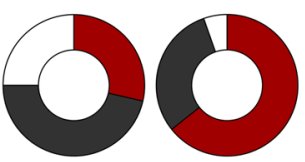

DNA / Centromeres / Tubulin

ATRi

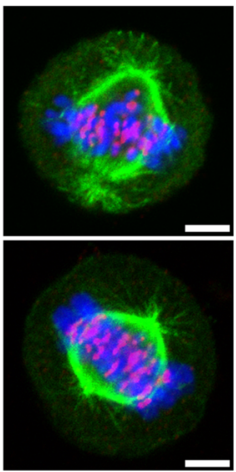

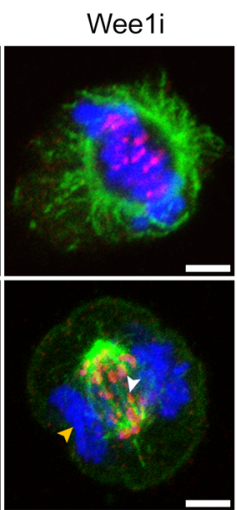

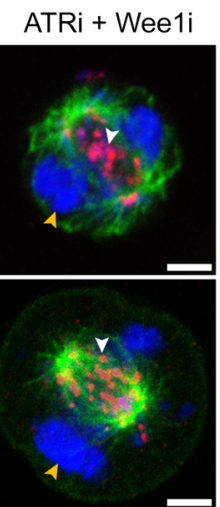

F

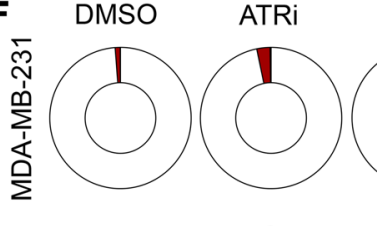

Wee1i ATRi + Wee1i

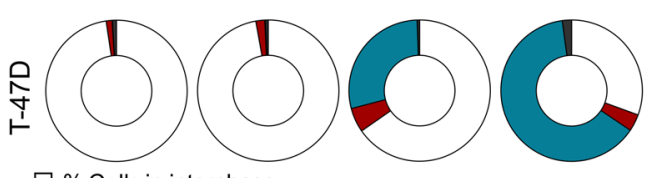

$\square \%$ Cells in interphase

$\square \%$ Cell death

$\%$ Centromere fragmentation

$\square \%$ Cells without centromere fragmentation

Figure 2. Combined ATR and Wee1 inhibition leads to mitotic defects and cancer cell death. (A-D) Live cell imaging of MDA-MB-231 expressing mCherry-histone H2B and GFP-tubulin. (A) Cells treated as indicated (ATRi $=1 \mu \mathrm{M} \mathrm{AZD6738,} \mathrm{Wee1i} \mathrm{=} 0.3 \mu \mathrm{M}$ AZD1775) were monitored by spinning-disk confocal microscopy. Representative images of cells following nuclear envelope breakdown (NEBD) are shown. (B) Quantification of the time from NEBD to anaphase. (C) Representative fates of 5 cells in the 4 treatment groups. (D) Quantification of observed cell fates $(n=56)$. Of note, when cell death occurred in interphase, the dying cells had previously undergone mitosis following drug addition. (E) Representative images of MDA-MB-231 or T-47D mitotic cells treated as in A. Fixed cells were stained for centromeres (red) and tubulin (green) by immunofluorescence and for DNA with DAPI (blue). Drug-induced clustering of centromeres (white arrows) spatially separated from the main mass of chromosomes (yellow arrow), a feature of centromere fragmentation, is clearly visible. Scale bars: $10 \mu \mathrm{m}$. (F) Quantification of cells that are in mitosis (red and blue) and display centromere fragmentation (blue) ( $n>1,000$ ), after fixing cells 4 hours after release from a double thymidine block in the presence of the indicated inhibitors. ${ }^{*} P<0.05,{ }^{* * *} P<0.0001$ (one-way ANOVA). 
A

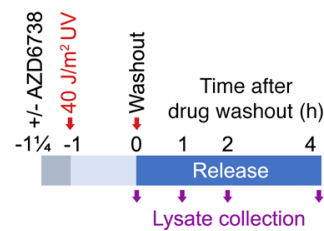

B

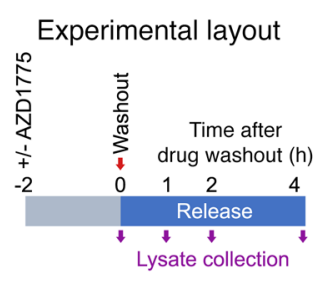

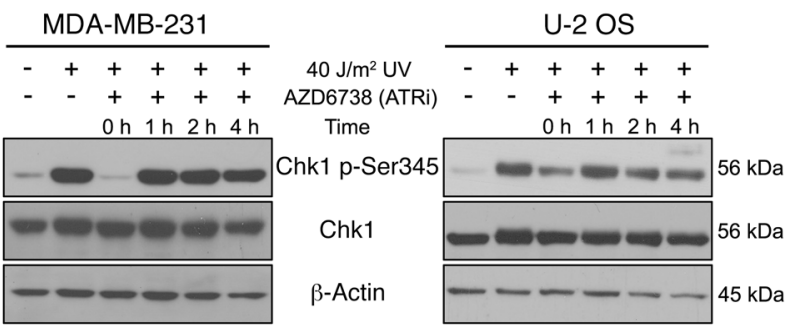

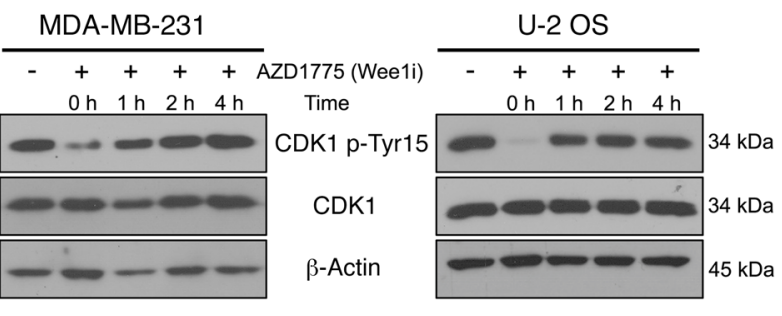

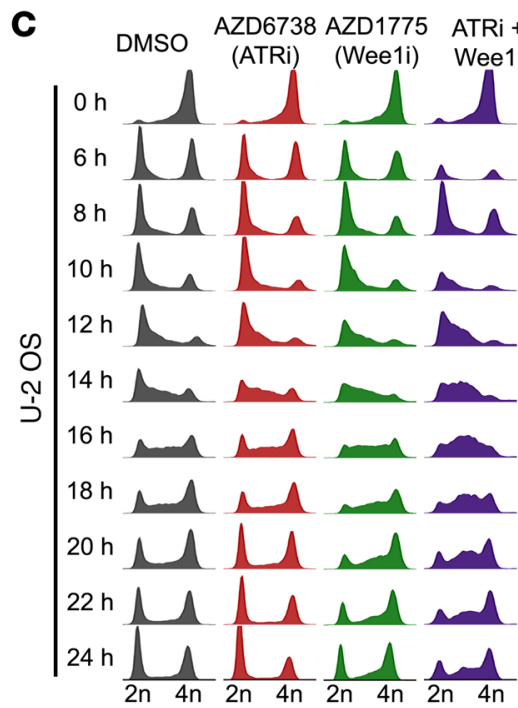
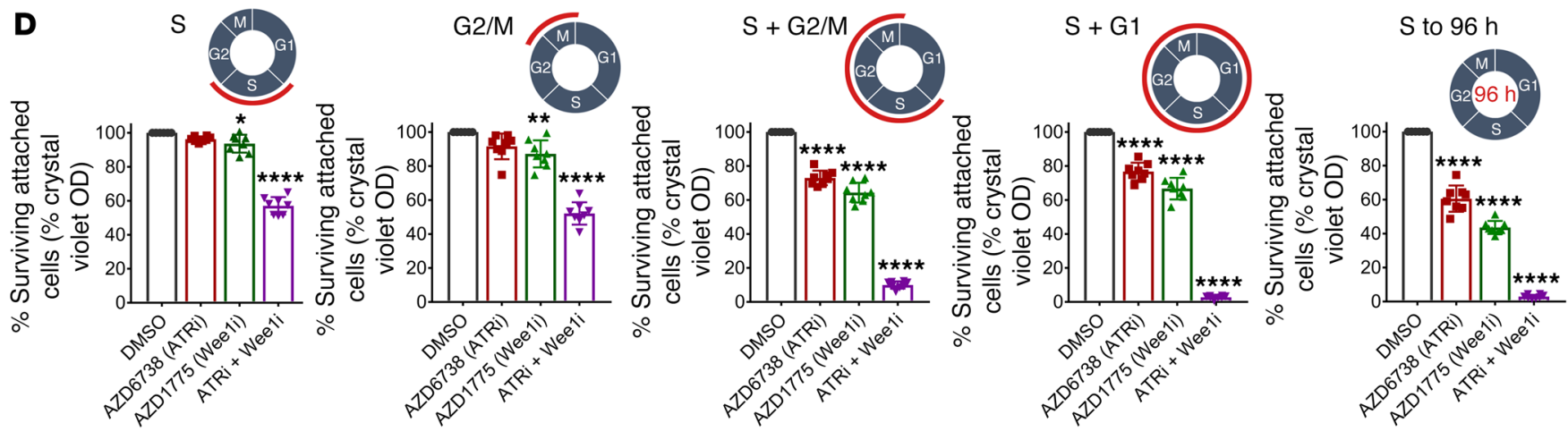

Figure 3. Contribution of cell cycle phases, during which ATR and/or Wee1 was inhibited, to overall cell killing. (A and B) AZD6738 and AZD1775 are reversible inhibitors. Immunoblots of MDA-MB-231 and U-2 OS cells treated as indicated. (A) The ATR inhibitor AZD6738 (1 $\mu$ M) was added to cells 15 minutes before irradiation with $40 \mathrm{~J} / \mathrm{m}^{2} \mathrm{UV}$, a strong activator of ATR. One hour after irradiation, AZD6738 was removed and the cells were washed and harvested at indicated times after drug removal. Restoration of ATR activity is observed 1 hour after AZD6738 washout. (B) Cells were incubated for 2 hours with $300 \mathrm{nM}$ Wee1 inhibitor AZD1775, leading to a strong reduction in phospho-CDK1. AZD1775 was then removed and cells washed, leading to restoration of Wee1 activity within 1-2 hours. (C) U-2 OS cells were synchronized by a thymidine-nocodazole block. Six hours after release, cells were treated with $1 \mu \mathrm{M}$ AZD6738 and/or 300 nM AZD1775. Cell cycle profiles were analyzed by propidium iodide staining and flow cytometry. (D) ATR and/or Wee1 in synchronized cancer cells were transiently inhibited with $1 \mu \mathrm{M}$ AZD6738 and/or $300 \mathrm{nM}$ AZD1775 during the indicated cell cycle intervals. Survival of drug-treated cells relative to vehicle control was measured after 4 days. Data represent mean $\pm \mathrm{SD}$. ${ }^{*} P<0.05,{ }^{* *} P<0.005$, and ${ }^{* * * *} P<0.0001$ (one-Way ANOVA).

in tumors, we established a human breast cancer xenograft model in mice. Because of the tumor (micro)environment, drugs that sensitize in vitro face additional challenges in selectively killing cancer cells in vivo. The different growth kinetics in vivo, hypoxia, intratumoral heterogeneity, interaction with the stroma, and of course drug delivery, influence efficacy. Moreover, side effects such as injury to normal tissues are of great concern.

We derived from MDA-MB-231, a triple-negative human breast cancer cell line (p53 mutated, BRCA wild type), a cell line that expresses the second-generation, less immunogenic firefly luciferase and the red-fluorescent protein tdTomato (36) (Supplemental Figure 12). In our orthotopic xenograft model, these MDA-MB-231-fluc2-tdTomato cells were injected into the fourth mammary fat pad of 6- to 8-week-old female NOD.Cg-Prkdc sid $I l 2 \mathrm{rg}^{\mathrm{tm} I \mathrm{Wl} l} / \mathrm{SzJ}$ (NSG) mice according to our approved animal proto$\mathrm{col}$ (AC16225). Once tumors reached a volume of $40-50 \mathrm{~mm}^{3}$ they were randomly allocated to treatment or vehicle arms. For our ini- tial biomarker study to validate in vivo inhibition of ATR and Wee1 by our inhibitors and to test DNA-damage induction in tumors, we administered $25 \mathrm{mg} / \mathrm{kg}$ AZD 6738 and $60 \mathrm{mg} / \mathrm{kg}$ AZD 1775 by oral gavage daily over 5 days. We harvested the tumors 1 hour after the last drug treatment (the approximate $\mathrm{T}_{\text {max }}$, when these drugs show maximal plasma concentrations; see ref. 37) (personal communication by AstraZeneca). Excised tumors ( $n=3$ mice per treatment group) were tested for ATR and Wee1 activity by immunohistochemistry, assessing phosphorylation of the respective Wee1 and ATR substrates CDK1 Y15 and ATR T1989 (Figure 4, A and B) (as all Chk1 p-S345 antibodies we tested did not work for immunohistochemistry, we used ATR autophosphorylation on Thr1989 as alternative readout for ATR activation; ref. 38 and see Supplemental Figure 6). Interestingly we not only confirmed ATR and Wee1 inhibition by AZD6738 and AZD1775, respectively, but also observed ATR activation in vivo in Wee1-inhibitor-treated tumors (Figure 4A). Our data also indicate that ATR or Wee1 inhibition 
A

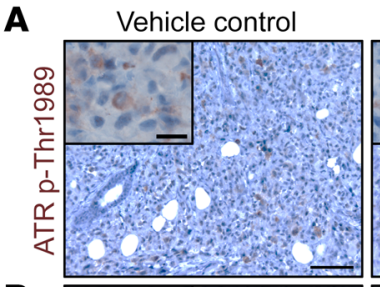

B

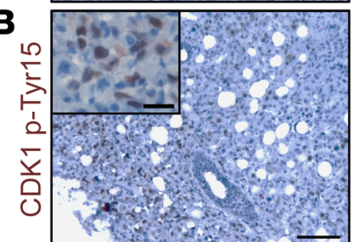

C

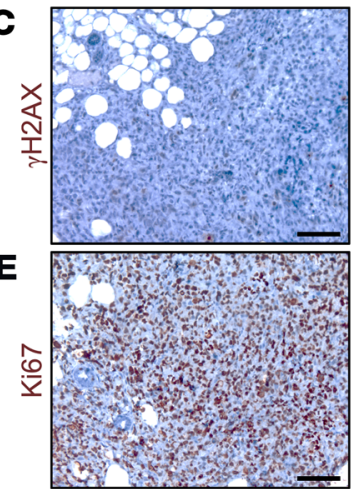

G
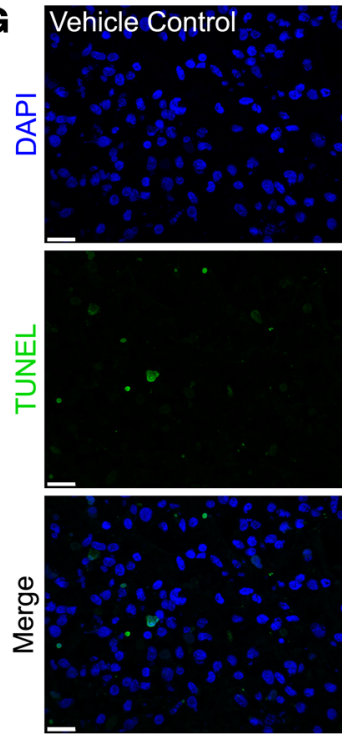

AZD6738 (ATRi)
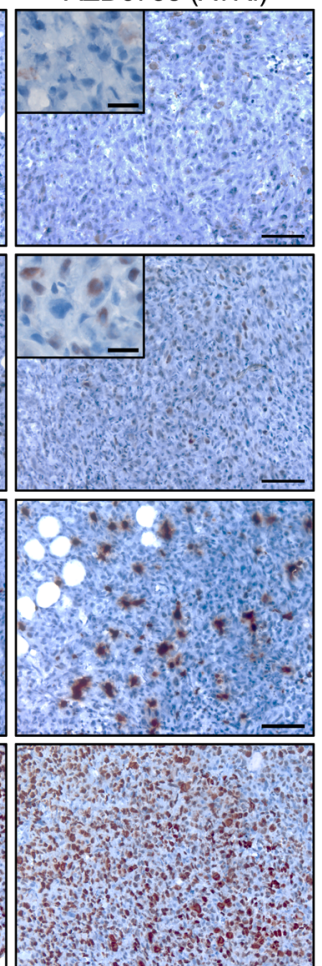

AZD6738 (ATRi)

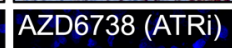

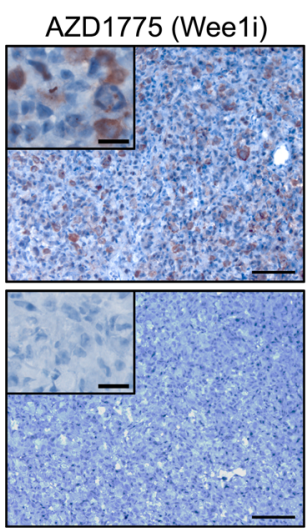

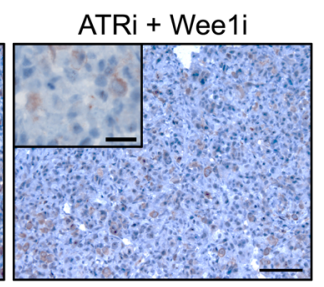

D
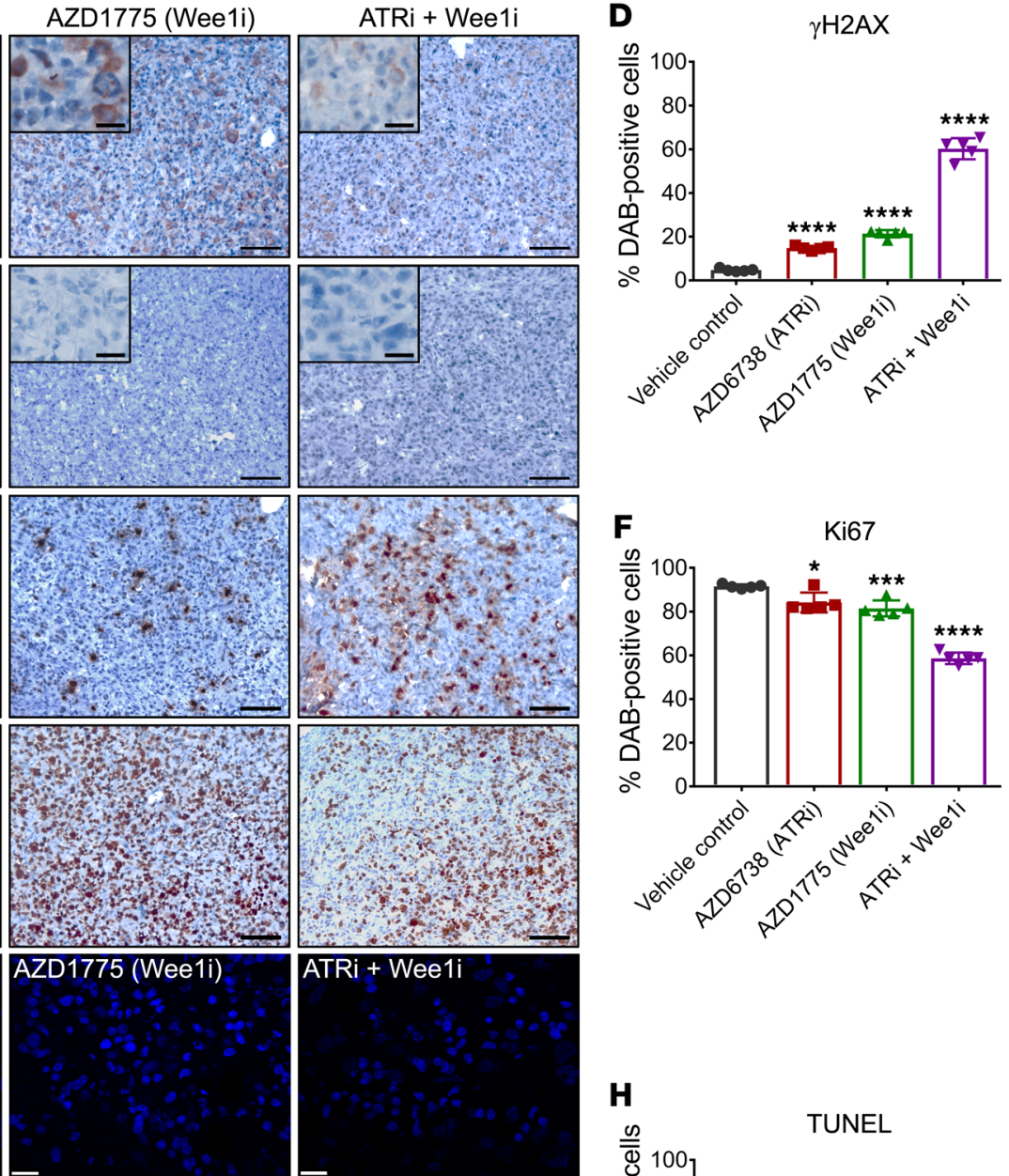

ATRi + Wee1i
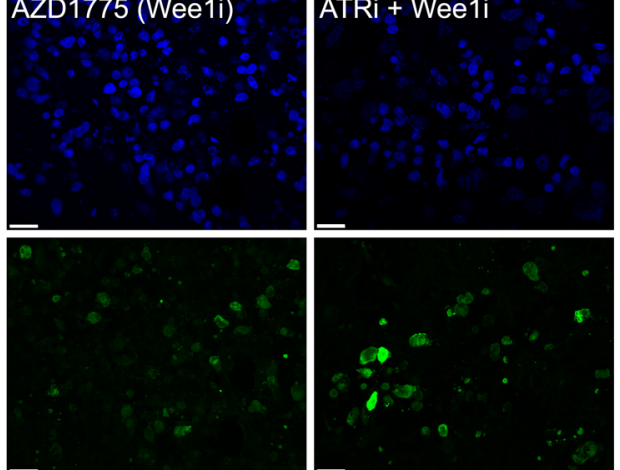

H

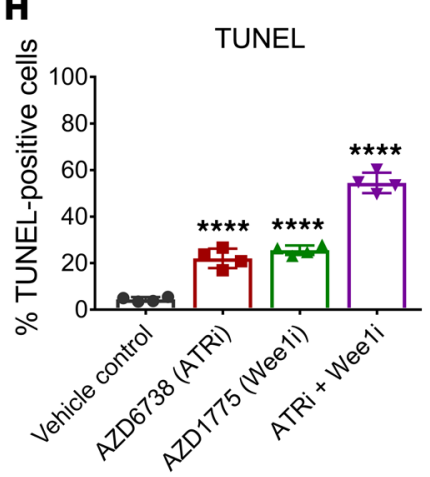

Figure 4. AZD6738 and AZD1775 inhibit ATR and Wee1, respectively, in vivo. MDA-MB-231-fluc2-tdTomato xenografts were excised for immunohistochemistry 1 hour after the last administration of the inhibitors to the mice by oral gavage for 5 days (25 mg/kg AZD6738 and/or $60 \mathrm{mg} / \mathrm{kg}$ AZD1775 daily). ATR (A) and Wee1 activity (B) was tested by probing for phosphorylation of their respective substrates, ATR Thr1989 and CDK1 Tyr15 (insets show tumor tissue at $\times 40$ magnification). (C and D) DNA damage was tested for with antibodies against $\gamma \mathrm{H} 2 \mathrm{AX}$. (E and F) Ki67 staining was used as a readout for proliferating cells. Scale bars: $100 \mu \mathrm{m}$ and $25 \mu \mathrm{m}$ (insets). (G and H) TUNEL assay was used to quantify cell death in excised tumor sections. Scale bars: $20 \mu \mathrm{m}$. Data represent mean \pm SD. ${ }^{*} P<0.05,{ }^{* *} P<0.001$, and ${ }^{* * *} P<0.0001$ (one-way ANOVA). DAB $=3,3^{\prime}$-diaminobenzidine.

over the same period leads to a significant increase in tumor cells with DNA damage, assayed by $\gamma \mathrm{H} 2 \mathrm{AX}$ staining (Figure $4, \mathrm{C}$ and D). Of note, combination treatment with the 2 kinase inhibitors seems to synergistically enhance the number of cells staining for $\gamma \mathrm{H} 2 \mathrm{AX}$ in the tumor $(P<0.0001$, one-way ANOVA) (Figure $4 \mathrm{D})$. It also reduces the fraction of proliferating tumor cells, as measured by Ki67 staining $(P<0.0001$, one-way ANOVA) (Figure $4, \mathrm{E}$ and
F). We also note a significant increase in the number of apoptotic cells, as measured by an increase in the number of TUNEL-positive tumor cells $(P<0.001$, one-way ANOVA) (Figure $4, \mathrm{G}$ and $\mathrm{H})$.

Combined ATR and Wee1 inhibition is well tolerated. As the aim of the conditional synthetic lethality is to spare normal tissue, we studied potential toxicities in treated mice, particularly in tissues with rapidly proliferating cells and relying on stem cells for regen- 
eration. We first tested tumor-bearing immunocompromised NSG mice ( $n=9$ per group), used for our xenografts, and immunocompetent C57BL/6 mice ( $n=6$ per group) for rudimentary indicators of side effects after treatment with $25 \mathrm{mg} / \mathrm{kg}$ AZD6738 and/or $60 \mathrm{mg} / \mathrm{kg}$ AZD1775 daily over a period of 26 days (Figure $5 \mathrm{~A})$. None of the mice showed significant changes in body weight (Figure 5, B and C), behavior (including food intake), or fecal consistency (data not shown). Postmortem analyses revealed no signs of inflammation or changes in spleen size. Only 1 mouse (in the ATR-alone treatment group) showed signs of partial hair loss (Supplemental Figure 13).

Owing to renewal kinetics, tissues relying on rapidly proliferating cells are particularly endangered by drugs that increase replication stress. Intestinal crypt stem cells support the continuous regeneration of the small intestinal epithelium, the most rapidly self-renewing tissue in adult mammals (39). We therefore examined intestinal cells for DNA damage and measured the villi length of ilea from NSG mice. Because of abrasion, villi are constantly replenished by the progenitor cells sitting in the crypt. Although we saw an increase in crypt cells staining for $\gamma \mathrm{H} 2 \mathrm{AX}$ (Figure 5D) in mice treated with Wee1 inhibitor alone or in combination, but not with ATR inhibitor alone, no treatment group showed a decrease in villi length by day 26 (Figure 5E). In mice, crypt stem cell depletion, e.g., by ionizing radiation, can lead to observable changes in villi within 4 days (40), yet the combination treatment over a period of 26 days is well tolerated in our mouse intestines, in agreement with no signs of diarrhea or changes in body weight. To test for changes in another tissue sensitive to genotoxic stress, we isolated the bone marrow from immunocompetent C57BL/6 mice after 26 days of inhibitor treatment. Bone marrow injury is one of the most common dose-limiting adverse effects of cancer therapy with genotoxic agents. Radiation and chemotherapy induce hematopoietic cell apoptosis, particularly in multipotent progenitor and hematopoietic progenitor cells, which proliferate and have lower DNA-repair capacity than the quiescent hematopoietic stem cells they derive from (41). Hematopoietic stem and progenitor cells can be identified by surface markers $(42,43)$. We used flow cytometry to quantify stem and progenitor cells from bone marrow using 2 different marker combinations, CD117 ${ }^{+}$ $\mathrm{Sca}^{+}$(hematopoietic stem and multipotent progenitor cells) (Figure 5, F and G) and $\mathrm{CD}^{2} 17^{+} \mathrm{Lin}^{-}$(which additionally include myeloid progenitor cells) (Figure 5, H and I) (41). We did not see any significant changes in the percentage of these subpopulations in bone marrows from inhibitor-treated mice compared with control mice (Figure 5, G and I).

To more rigorously test for drug-induced damage to normal tissue we harvested additional tissues from tumor-bearing immunocompromised NSG mice ( $n=3$ per group) and immunocompetent C57BL/ 6 mice ( $n=3$ per group) immediately after the 26 -day drug treatment, or 1 (33 days) or 2 weeks after the last day of drug administration (Figure 6 and data not shown). Only in the ilea and spleens of mice at the end of the drug treatment did we observe an increase of $\gamma \mathrm{H} 2 \mathrm{AX}$-staining cells. No DNA damage was observable in lungs, kidneys, or livers from either NSG or C57BL/6 mice (Figure 6 and Supplemental Figures 7-11). Remarkably, after a period of just 7 days after the last drug administration, the number of DNA-damaged cells in the ilea or spleens returned to background levels (Figure 6). We also analyzed the blood of mice at the end of, and 1 or 2 weeks after, drug treatment. Pathological evaluations did not reveal any significant changes in complete blood cell count (Supplemental Tables 1 and 2), in agreement with the lack of observable hematopoietic stem cell depletion (Figure 5, F-I). In summary, the increased endogenous DNA damage in cancer cells compared with even actively proliferating normal cells seems to provide a significant therapeutic window for the combination treatment.

Combined inhibition of ATR and Wee1 leads to tumor remission, increased survival, and inhibition of metastasis. To test drug efficacy in longitudinal studies using our xenograft model, once tumors reached a volume of $40-50 \mathrm{~mm}^{3}$ mice were randomly allocated to treatment or vehicle arms ( $n=9$ mice per treatment group). These mice were administered $25 \mathrm{mg} / \mathrm{kg}$ AZD6738 and/or $60 \mathrm{mg} / \mathrm{kg}$ AZD1775 daily over a period of 26 days (Figure 5A, yellow shades in Figure 7, B, C, and F) and tumor growth was followed by caliper measurement every second day and metastasis by weekly inspection with a bioluminometer (Figure 7A). We observed significant inhibition of tumor growth $(P<0.0001$, two-way ANOVA) (Figure 7B) by treatment with AZD6738 and AZD1775. While treatment with AZD6738 or AZD1775 alone delayed tumor growth, tumor expansion resumed rapidly when drug treatment was stopped. However, combination treatment led to tumor shrinkage to below $1 \mathrm{~mm}^{3}$, and in 6 of 9 cases even to complete remission, as determined by impalpable tumor levels. Although we have not observed complete eradication so far (the high sensitivity of bioluminescence allows for the visualization of residual MDA-MB231-fluc2), we speculate that a proportionate level of cell killing in immunocompetent patients could lead to tumor control. In our immunocompromised NSG mice that have been treated with the inhibitor combination, tumors do eventually recur (Supplemental Figure 14). Nevertheless, and although mice were only treated for 26 days, mice treated with AZD6738 plus AZD1775 lived significantly longer $(P<0.0001$, log-rank Mantel-Cox test $)-$ median survival after start of treatment: AZD6738, 60 days; AZD1775, 62 days; AZD6738+AZD1775, 103 days; vehicle control treated, 53 days (Figure 7C) - paralleling the cancer-selective synthetic lethality observed in vitro.

Tagging MDA-MB-231 cells with firefly luciferase also allowed us to follow metastasis by noninvasive bioluminescence imaging. As can be seen by representative images of mice at week 7 (16 days after the last drug administration) and the statistical analysis of bioluminescence at distant sites, combined Wee1 and ATR inhibition strongly suppressed metastasis (Figure 7D). While Wee1 or ATR inhibitor stand-alone treatment did not show any significant inhibition of metastasis, bioluminescence levels at distant sites in the combination treatment are below the background threshold $(P=0.0383$, one-way ANOVA) (Figure 7E).

To further investigate inhibition of metastasis we treated a set of mice ( $n=4$ mice per treatment group) only when tumors reached a volume of approximately $250 \mathrm{~mm}^{3}$. At that point micrometastasis should already have occurred, as the corresponding time relates to approximately 4-5 weeks later in tumor growth compared with the previous experiments (compare tumor volumes and metastasis at week 7 for control mice in Figure 7, B and D). Again, mice were randomly allocated to treatment arms, consisting of 
A

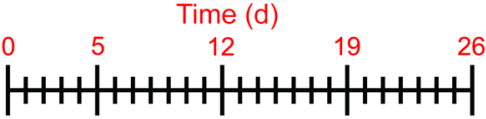

25 mg/kg AZD6738 and/or 60 mg/kg AZD1775

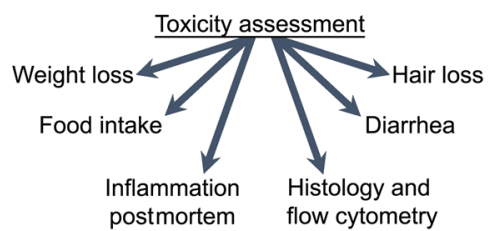

D

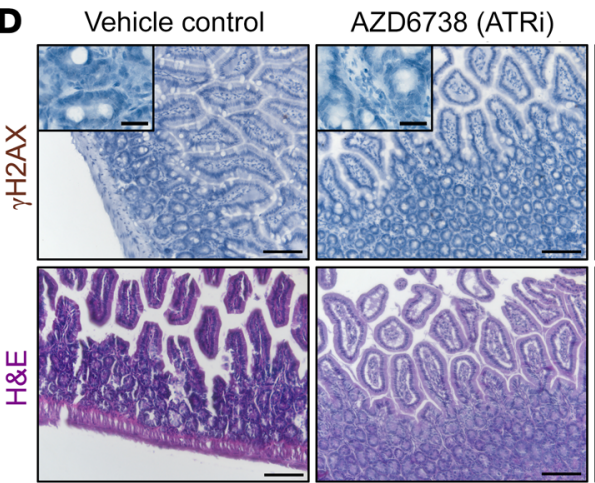

B

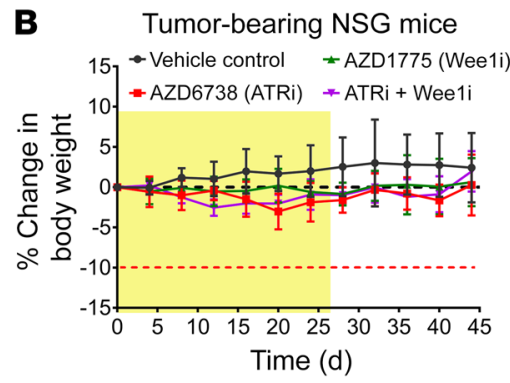

AZD1775 (Wee1i)

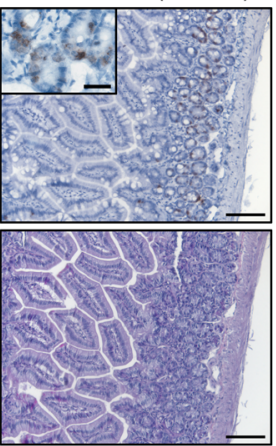

ATRi + Wee1i

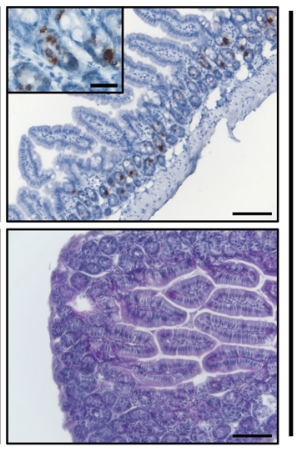

\section{F}

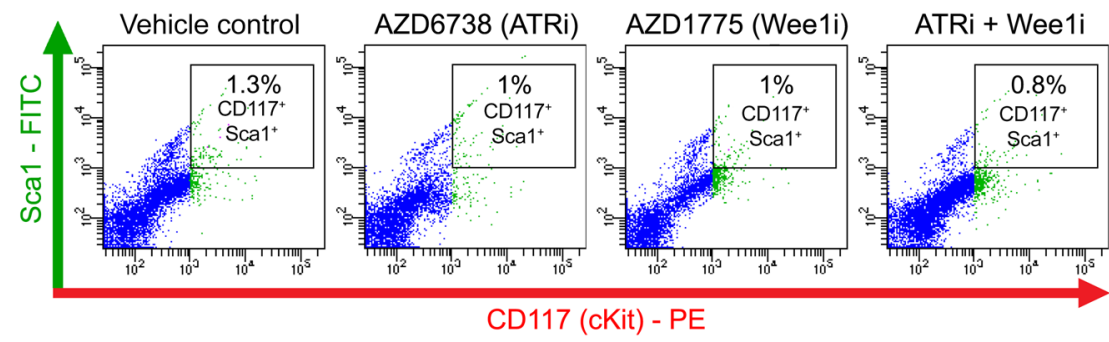

H

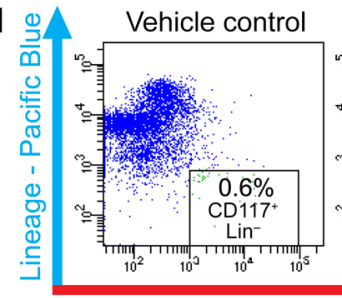

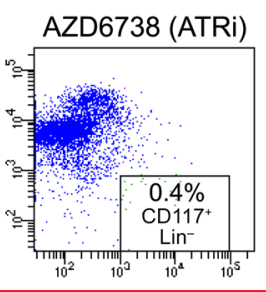

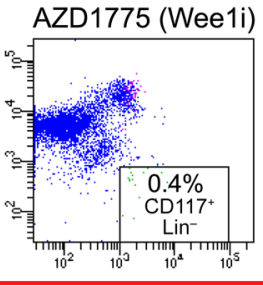

CD117 (cKit) - PE

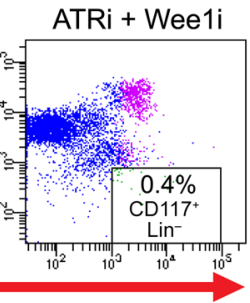

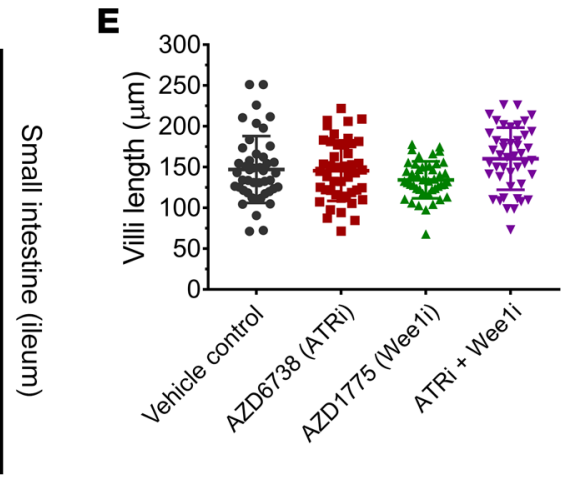

E
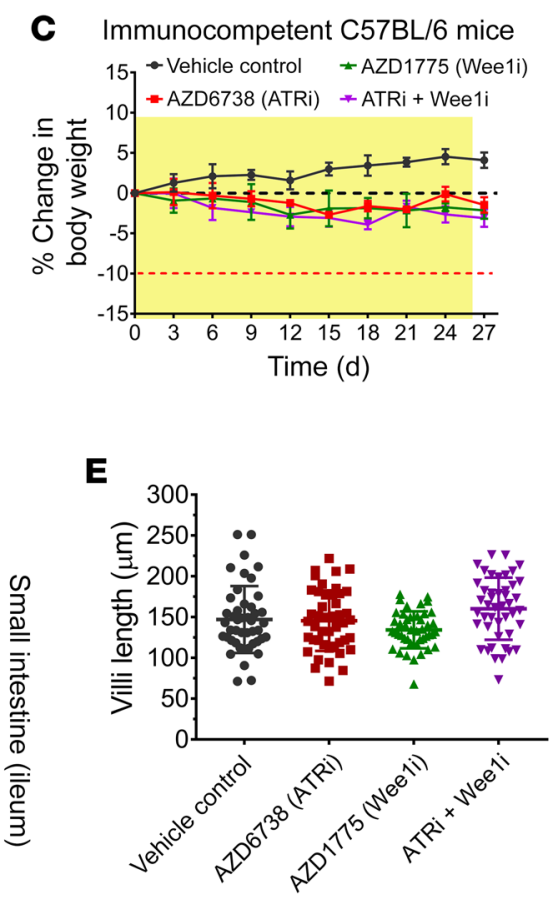

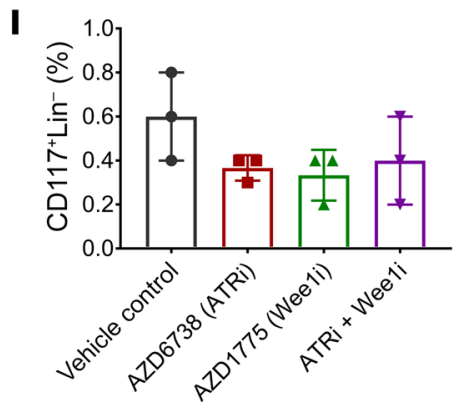

Figure 5. Combination treatment with ATR and Wee1 inhibitors and normal tissue toxicity. (A) Mice were treated for 26 days daily with 25 mg/ kg AZD6738 and/or 60 mg/kg AZD1775 and tested for adverse effects. (B and C) No significant body weight changes are observed in tumor-bearing immune-deficient NSG or in immunocompetent C57BL/6 mice. (D and E) Although Wee1 inhibition leads to some $\gamma \mathrm{H} 2 \mathrm{AX}$ staining in the crypts of NSG mouse ilea (see insets) (D), no significant change in villi length is observed (E). $n=50$ refers to 50 measurements in each of 3 mice per group. Scale bars: $100 \mu \mathrm{m}$ and $25 \mu \mathrm{m}$ (insets). (F-I) No significant depletion of hematopoietic stem and progenitor cells isolated from treated C57BL/6 mice is observed. Bone marrow cells were isolated from C57BL/6 mice treated as described in $\mathbf{A}$ and analyzed with the indicated surface markers by flow cytometry. (F and G) Hematopoietic stem and multipotent progenitor cells stained for CD117 and Sca1. (H and I) The CD117+ Lin ${ }^{-}$population additionally includes myeloid progenitor cells. Data represent mean \pm SD.

a 26-day period of daily administration of $25 \mathrm{mg} / \mathrm{kg}$ AZD6738 and/or $60 \mathrm{mg} / \mathrm{kg}$ AZD1775. As seen before, AZD6738 and AZD1775 single treatment led to tumor growth delay $(P<0.0001$, two-way ANOVA), but combined treatment led to tumor shrinkage
( $P<0.0001$, two-way ANOVA) (Figure 7F). Secondary tumors were observed in the thoracic lymph nodes of control, AZD6738-, or AZD1775-treated mice, but not in animals receiving the combination treatment ( $P=0.0061$, one-way ANOVA) (Figure 7G). 

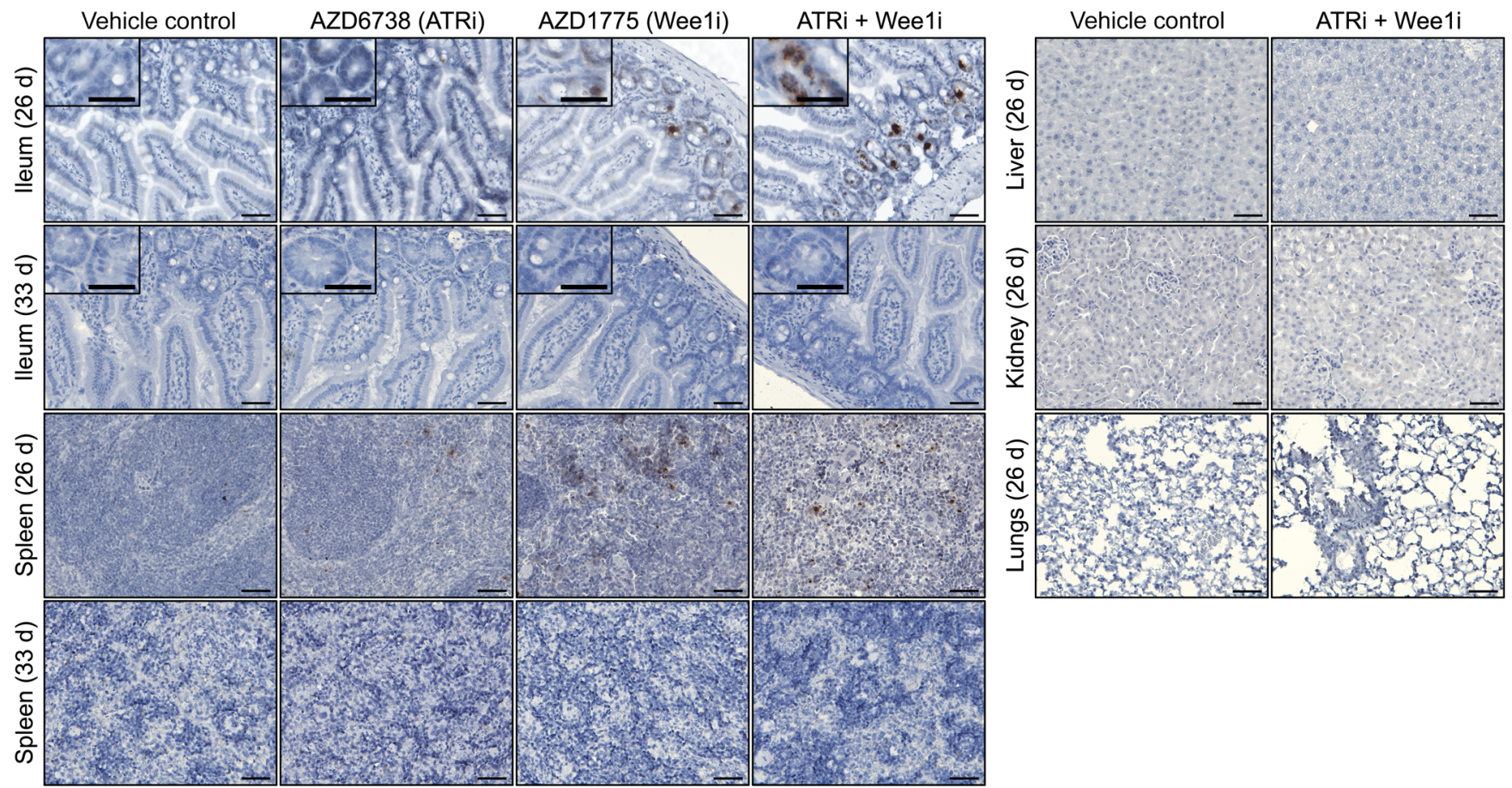

Figure 6. Evaluation of normal tissue DNA damage. Tissues from tumor-bearing NSG mice (or immunocompetent C57BL/6 mice without tumors, shown in Supplemental Figure 11) were harvested on the last day (26 d) or 1 week after (33 d) the last day of a 26-day treatment period with AZD6738 and/or AZD1775. While lung, liver, and kidney did not show any signs of DNA damage, some cells in the ileum and spleen were found to stain for $\gamma \mathrm{H} 2 \mathrm{AX}$ at the end of the treatment ( $26 \mathrm{~d})$. However, 1 week later ( $33 \mathrm{~d}$ ), ilea and spleens recovered from the drug treatment, as measured by staining for $\gamma \mathrm{H} 2 \mathrm{AX}$. Scale bars: $25 \mu \mathrm{m}$ and $20 \mu \mathrm{m}$ (insets).

Even more compelling, tissues from the mice euthanized at the end of the treatment were inspected for micrometastases by bioluminescence, a technique that allows us to detect clusters of as few as 20 cells. Unlike in the case of control or single-inhibitor-treated mice, which showed metastasis to lymph nodes, lungs, liver, bone, gut, and in some case also to the brain and ovaries, tissues from combined ATR- and Wee1-inhibitor-treated animals showed no detectable micrometastases (Table 2).

ATR and Wee1 activity are critical for breast cancer stem cell survival. Our observation that combined ATR and Wee1 inhibition suppresses metastasis of highly invasive MDA-MB-231 (Figure 7, $\mathrm{D}-\mathrm{F}$, and Table 2) could be explained by inhibition of the process of metastasis per se or a depletion of cells able to spread and to initiate tumors at distant sites. Breast cancer stem cells have been implicated in metastasis because of their high cellular plasticity, enabling them to undergo epithelial-mesenchymal transition, and their tumor initiating potential. This prompted us to isolate a subpopulation enriched in cancer stem cells from cell lines of 2 different breast cancer subtypes, MCF7 (luminal B) and MDA-MB-231 (claudin low), by their dye efflux propensity (44) (Figure 8A). Cancer stem cells often show upregulation of transporter proteins in the ATP-binding cassette family, such as ABCG2. Confirming the stem cell character of the isolated subpopulation, a much lower number of seeded cells from the fraction with high dye efflux capacity (side population, SP) is required to form mammospheres than cells with low efflux capacity (non-side population, NSP) (Table 3 and Figure 8B). We next compared cooperative cell killing by ATR and Wee1 inhibitors in the cancer stem cell-enriched SP to an NSP. MCF7 and MDA-MB-231 cancer stem cells (SP) are more resistant than the control subpopulation (NSP) to either AZD6738 or AZD1775 alone, but surprisingly showed similar sensitivities to the combined treatment (Figure 8, C and D). This unexpected finding is due to higher synergistic effects in cancer stem cells than in cancer cells without stem cell features (e.g., lower Bliss CIs at 300 nM AZD6738 and 100 nM AZD1775: 0.40 versus 0.90 for MCF7 and 0.41 versus 0.75 for MDA-MB-231). To our knowledge this is the first reported observation of increased synergistic effects of cytotoxic agents in cancer stem cells compared with bulk cancer cells. The increased synergy in cancer stem cells, although they are more resistant to the single agents, could explain the strong antimetastatic effect by the combination treatment observed in our animal model.

\section{Discussion}

Tumor heterogeneity constitutes one of the biggest barriers to effective cancer therapies. Therapies merely targeting the bulk of cancer cells are often destined to fail because induced clonal drifts and the formation of dormant cells decrease tumor control probability. Furthermore, activation of alternative pathways to counteract targeted therapies can lead to drug resistance. Here, we describe a strategy designed to take advantage of the cancerintrinsic property of DNA damage (8), a feature shared by all clones (albeit potentially to different extents). Genomic instability is a driver of tumorigenesis and has been designated as a hallmark of cancer (45). Cancer cells typically show oncogene-driven genomic changes such as an aberrant number or structure of chro- 
A

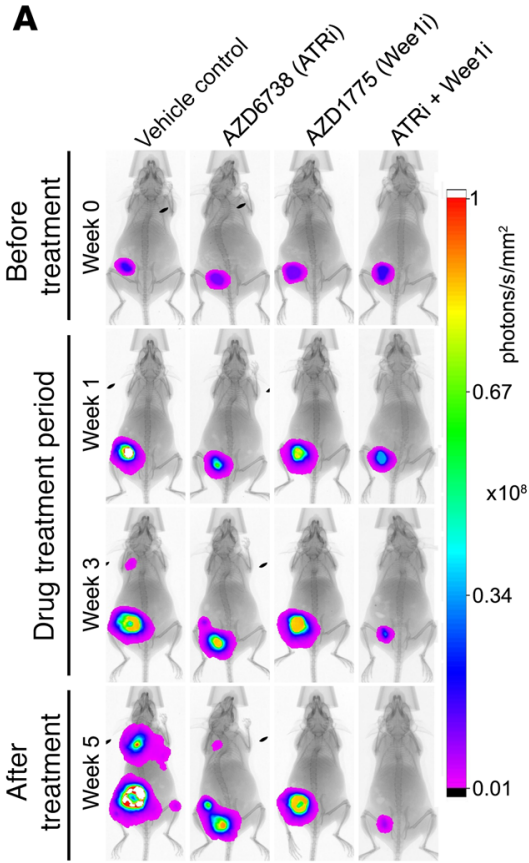

B

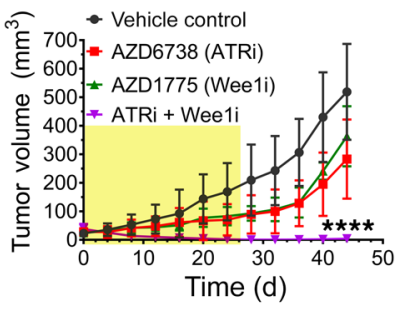

D

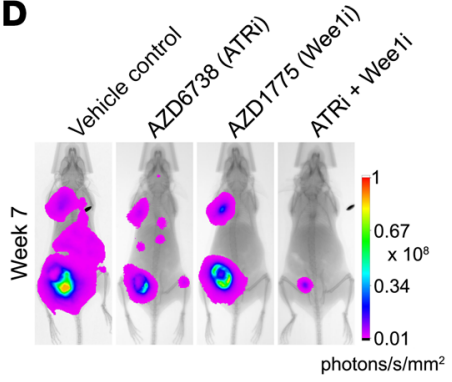

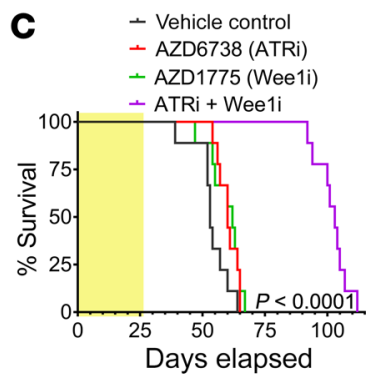

E

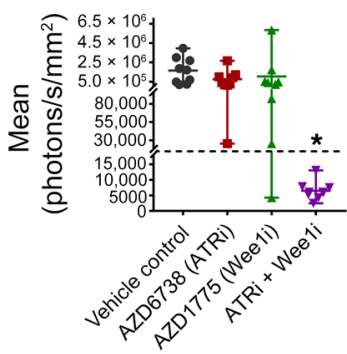

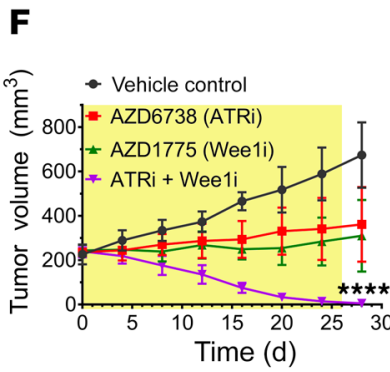

G

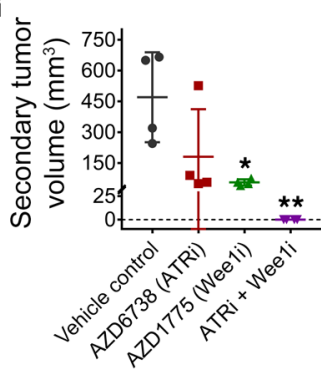

Figure 7. Combination treatment with ATR and Wee1 inhibitors and tumor control. (A-E) NSG mice were injected orthotopically with MDA-MB-231-fluc2tdTomato-labeled breast cancer cells and treated for 26 days (indicated by yellow shades) with 25 mg/kg AZD6738 and/or 60 mg/kg AZD1775 after tumors reached approximately $40 \mathrm{~mm}^{3}$. (A) Tumor progression was monitored weekly by bioluminescence imaging. (B) Tumor growth of mice in the 4 treatment arms ( $n=9$ per group). (C) Kaplan-Meyer survival curves of treated mice ( $n=9$ per group). (D and E) Metastasis in regions distal to the primary tumor was assessed 7 weeks after treatment initiation ( $n=9$ per group). The dotted line indicates background threshold (E). (F and $\mathbf{G})$ To further investigate inhibition of metastasis, a group of MDA-MB-231-fluc2-tdTomato tumors ( $n=4$ per group) were allowed to grow to approximately 250 mm ${ }^{3}$ before treatment as in A. Combination treatment leads to tumor shrinkage (F). Unlike control or single-agent-treated mice, those treated with AZD6738 and AZD1775 had no detectable secondary tumors (G). Data represent mean \pm SD. ${ }^{*} P<0.05,{ }^{*} P<0.01,{ }^{* * *} P<0.0001$ by 2 -way ANOVA (B and F), log-rank Mantel-Cox test (C), or 1-way ANOVA (E and G).

mosomes (chromosomal instability), microsatellite instability, and/or the mutagenic load. While the impairment of checkpoints that should prevent these events are drivers of tumorigenesis, the increase in accumulated DNA damage leads to replication stress (46) and a high risk of mitotic failure, making the survival of cancer cells heavily reliant on an often partially defective DDR.

The gene products of ataxia telangiectasia mutated (ATM) and ATR are apical kinases of pathways activated by DNA damage. Unlike ATM, which is frequently lost in cancers (47), ATR is an essential gene for the $\operatorname{DDR}(21,22)$ and ATR activity is often upregulated in cancer cells $(48,49)$. ATR activation is important for DNA-damage repair by homologous recombination (10-12). Furthermore ATR (via Chk1) together with Wee1 negatively regulates the activity of CDK2 and especially of CDK1, the only essential CDK in mammals (50). Likely owing to the reliance of cancer cells on the G2/M checkpoint to protect them from mitotic catastrophe as a consequence of excessive DNA damage, Wee1 was found upregulated in several cancer types (51). The importance that Wee1 and ATR were found to have in cancer cell survival make them attractive therapeutic targets.

A model for the synergistic cell killing by ATR and Wee1 inhibition. Here, we report cancer-selective synergistic killing by ATR and Wee1 inhibition. (While this manuscript was in preparation, another group reported synergistic killing of triple-negative breast cancer cells by Wee1 and ATR inhibitors; ref. 52). Our data support a model in which synergistic killing by ATR and
Wee1 inhibitors is triggered by Wee1 inhibition-induced DNA damage during replication, abrogation of ATR-mediated S phase checkpoint activation, inhibition of ATR-dependent homologous recombination, and amplified by increased entry into mitosis with defective genomes due to combined inhibition of ATR and Wee1 (Supplemental Figure 15). High replication stress in cancer cells could be due to the high level of baseline DNA damage per se, but also to the resulting exhaustion of factors needed for both repair

\section{Table 2. Metastases in animals treated with ATR and Wee1} inhibitors

$\begin{array}{lcccc}\text { Tissue } & \begin{array}{c}\text { Vehicle } \\ \text { control }\end{array} & \begin{array}{c}\text { AZD6738 } \\ \text { (ATRi) }\end{array} & \begin{array}{c}\text { AZD1775 } \\ \text { (Wee1i) }\end{array} & \begin{array}{c}\text { ATRi + } \\ \text { Wee1i }\end{array} \\ \text { Lungs } & 4 / 4 & 4 / 4 & 4 / 4 & 0 / 4 \\ \text { Lymph nodes } & 4 / 4 & 4 / 4 & 4 / 4 & 0 / 4 \\ \text { Bone } & 4 / 4 & 2 / 4 & 1 / 4 & 0 / 4 \\ \text { Liver } & 4 / 4 & 4 / 4 & 4 / 4 & 0 / 4 \\ \text { Brain } & 2 / 4 & 1 / 4 & 0 / 4 & 0 / 4 \\ \text { Gut } & 4 / 4 & 4 / 4 & 4 / 4 & 0 / 4 \\ \text { Ovaries } & 4 / 4 & 3 / 4 & 1 / 4 & 0 / 4\end{array}$

Ex vivo bioluminescence imaging of excised tissues revealed micrometastases in several organs from control or single-inhibitor-treated mice, but no micrometastases were observed in the AZD6738/AZD1775 combination treatment group. 

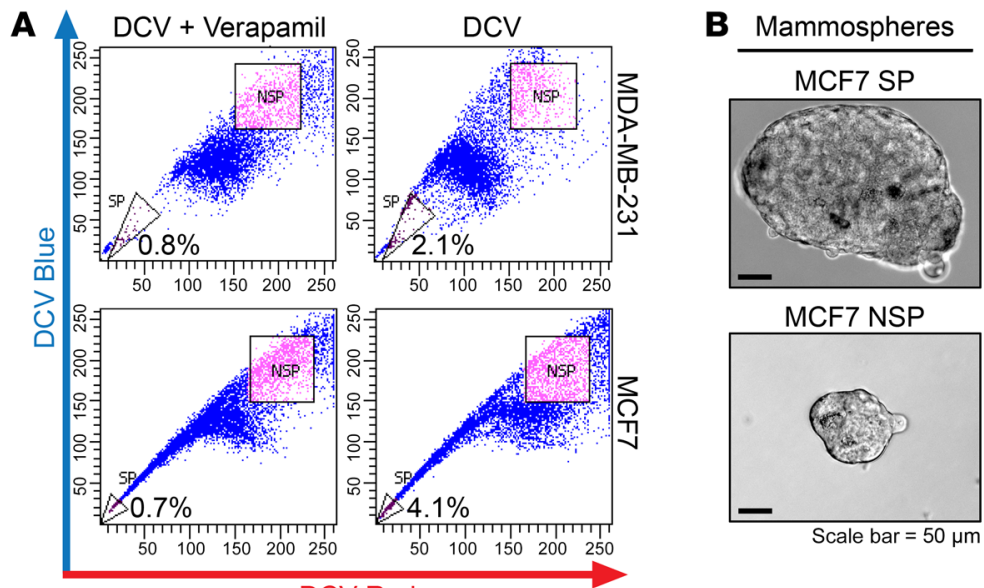

MCF7 NSP

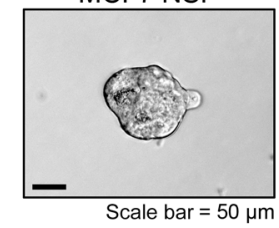

DCV Red

C

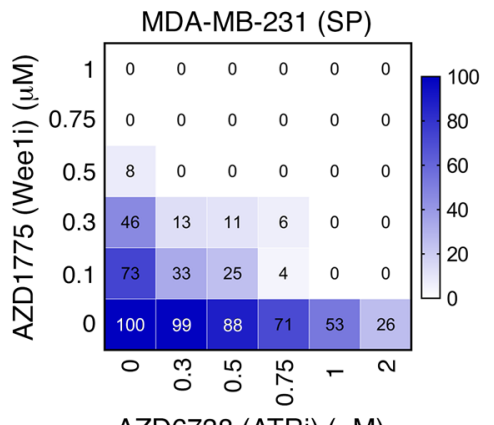

AZD6738 (ATRi) ( $\mu \mathrm{M})$

D

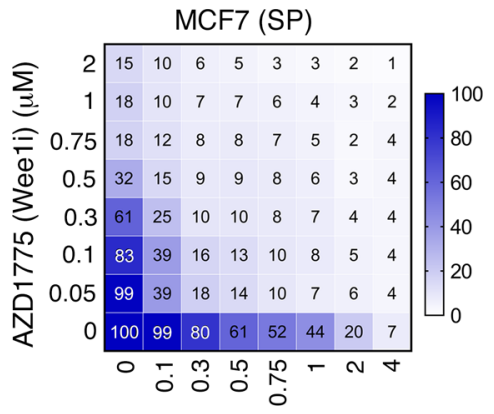

AZD6738 (ATRi) $(u \mathrm{M})$

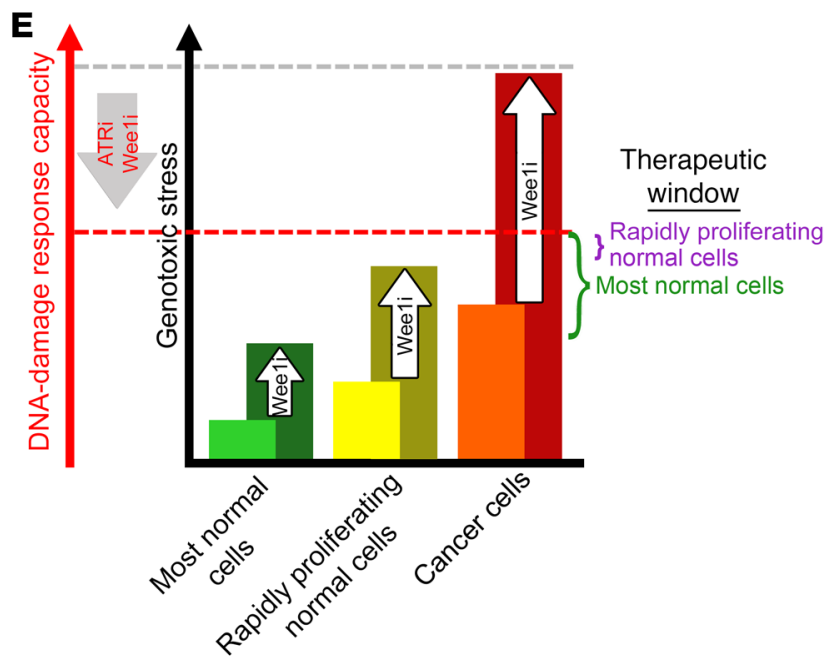

F

F

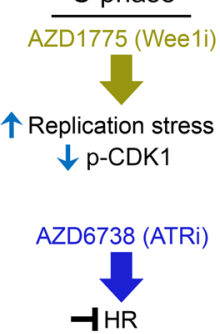

$\downarrow \mathrm{p}-\mathrm{CDK} 1$

* Checkpoint abrogation

Cells exit S-phase with damaged DNA
Figure 8. Synergistic killing of breast cancer stem cells by ATR and Wee1 inhibitors. (A) Isolation of cancer stem cell-enriched subpopulations (side population, SP) from MDA-MB-231 or MCF7 based on their increased dye (DyeCycle Violet, DCV; see supplemental methods) efflux properties. Verapamil, an inhibitor of drug efflux pump proteins, particularly of the $A B C$ transporter family, served as negative control. (B) Isolated SPs show an increased ability to form mammospheres compared with control subpopulations (non-side population, NSP). Representative images of mammospheres are shown. (C and D) Four-day survival assays of cancer stem cellenriched SPs and control cells (NSPs) isolated from MDA-MB-231 (C) and MCF7 (D). Plated cells were treated with indicated concentrations of AZD1775 and/or AZD6738. Color bars indicate percentage survival normalized to untreated cells. (E) Model of cancer-selective synergistic cell killing by combined ATR and Wee1 inhibition. Cancer cells have higher baseline levels of genotoxic stress than normal cells. Wee 1 inhibition increases genotoxic stress, while ATR and Wee1 inhibition together lower cellular DNA-damage response capacity (in the simplified model to the same extent, but potentially higher in cancer cells relying on these 2 kinases for survival). A therapeutic window is created for the selective killing of cancer cells. (F) Cell cycle-dependent effects of ATR and Wee1 inhibition contributing to overall cell death following mitotic catastrophe. HR, homologous recombination.

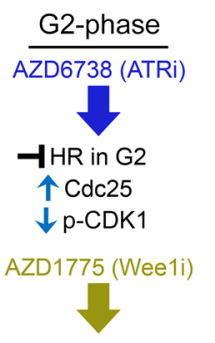

* CDK1 is not phosphorylated

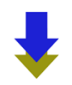

Cells enter M-phase with damaged DNA
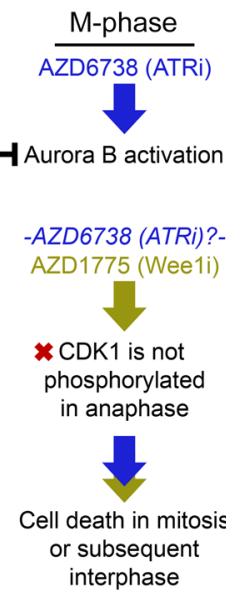
Table 3. Mammosphere-forming capabilities of SP and NSP cells

\begin{tabular}{|cccc} 
Cells plated & $\begin{array}{c}\text { MDA-MB-231 } \\
\text { per well }\end{array}$ & $\begin{array}{c}\text { No. of wells positive } \\
\text { for mammospheres }\end{array}$ & $\begin{array}{c}\text { No. of wells positive } \\
\text { for mammospheres }\end{array}$ \\
\hline Side Population (SP) & 1 & $0 / 6$ & $2 / 6$ \\
\hline & 2 & $0 / 6$ & $2 / 6$ \\
\hline Non-Side Population (NSP) & 5 & $0 / 6$ & $3 / 6$ \\
& 10 & $0 / 6$ & $4 / 6$ \\
& 100 & $5 / 6$ & $5 / 6$ \\
& 1,000 & $6 / 6$ & $6 / 6$ \\
& 2 & $0 / 6$ & $0 / 6$ \\
& 5 & $0 / 6$ & $0 / 6$ \\
& 10 & $0 / 6$ & $0 / 6$ \\
& 100 & $0 / 6$ & $0 / 6$ \\
\hline & 1,000 & $1 / 6$ & $0 / 6$ \\
\hline
\end{tabular}

Isolated side population cells demonstrate higher mammosphere-forming capabilities as compared with the non-side population cells. and others, Chk1 inhibition is not phenotypically identical to ATR inhibition $(10,25,55)$.

Cancer-selective synthetic lethality, tumor remission, and inhibition of metastasis. Our preclinical mouse data indicate that at doses leading to strong tumor shrinkage, combined ATR and Wee1 inhibition shows minimal adverse effects. The absence of diarrhea or villi change in the ilea as well as of a significant loss of hematopoietic stem and progenitor cells, indicators of intestinal damage or bone marrow injury respectively, suggest that tissues relying on rapidly proliferating cells for homeostasis are less sensitive to the combination treatment than tumor cells. Phase I studies of AZD6738 (as well as other ATR inhibitors) are currently being undertaken. AZD1775 (currently the only Wee1 inhibitor in clinical development) has already progressed to several phase II trials, usually in combination with genotoxic agents such as carboplatin or gemcitabine (55). The strong synergistic effects on tumor control described here, leading to complete remission in 6 of 9 cases by the AZD6738/ AZD1775 combination treatment, provide an ideal base and replication, such as RPA (53). ATR plays an essential role for cancer cells to survive replication stress. Already hypersensitive to ATR inhibition, we propose that Wee1 inhibition leads to even higher replication stress in cancer cells, making them unable to avoid DNA damage during replication at ATR-inhibitor doses tolerable to the animals (or patient). Highly proliferative normal tissues by contrast do not have such high baseline replication stress and can tolerate the combination treatment (Figure 8E). This model is supported by our observation that reversal of ATR or Wee1 inhibition alone following $S$ phase leads to minimal cell death (Figure 3D), indicating that the resulting increase in replication stress can be rescued by repair before entry into mitosis. Combined inhibition during replication on the other hand, even if reversed after S phase, leads to substantial cell killing, likely due to extensive genome damage that cannot be repaired before cells enter mitosis. Similarly, combined ATR and Wee inhibition after $\mathrm{S}$ phase completion leads to extensive cell death. This might be due to G2/M checkpoint abrogation and the consequent premature entry into mitosis with unrepaired endogenous DNA damage, but also to functions of ATR and Wee1 during mitosis. ATR was reported to contribute to faithful chromosome segregation by promoting Aurora B activation at centromeres (54). Also, Wee1 has a role in mitosis beyond regulating the G2/M checkpoint, as residual Wee1 (potentially together with ATR) inhibits CDK1 activity in anaphase, which controls mitotic exit (9). The abrogation of ATR and Wee1 activity during different phases of the cell cycle cooperatively leads to cell death caused by mitotic defects (Figure 8F). Cell death can occur in mitosis or in interphase after aborted or completed mitosis. As a consequence of coordinated effects that Wee1 and ATR have on faithful cell cycle progression, particularly in cells with high baseline DNA damage, a therapeutic window opens to lower the activity of these 2 kinases to levels lethal for cancer cells, but tolerable to normal tissues. This is in stark contrast to Chk1 inhibition, which - particularly when combined with Wee1 inhibition (Supplemental Figure 2) - shows high toxicity in nontransformed cells. As previously pointed out by us for phase I clinical trials. Even more striking is our observation that combined Wee1/ATR inhibition leads to a strong inhibition of metastasis. We observe both inhibition of tumor spread by a 26-day AZD6738/AZD1775 treatment started when tumors are still small as well as the absence of metastatic lesions following the same treatment in mice, when the treatment was initiated after micrometastasis has already happened (Figure 7, D-G, and Table 2). This observation could be explained by our surprising finding that breast cancer stem cell-enriched populations, although more resistant to either ATR or Wee1 inhibition alone, show a higher synergy in cell killing by cotreatment with AZD6738 and AZD1775 than bulk cells. Cancer stem cells from a variety of tissues were found to display elevated radiation resistance and chemoresistance (56). Interestingly, glioma, colon, and lung cancer stem cells were found to have a stronger ATR response to genotoxic agents than bulk cancer cells (57-59), and glioma stem cells were found to be more sensitive to Wee1 inhibition than neural stem cells (60). It could be that the reliance of cancer stem cells on ATR or Wee1 to withstand genotoxic insults makes them particularly vulnerable to combined Wee1/ATR inhibition. Because metastasis is the main cause of death in cancer patients, the antimetastatic activity and the propensity to kill cancer stem cells could make a combined AZD6738/AZD1775 regimen suitable for stand-alone treatment or for adjuvant therapy.

Potential strategies for patient selection. Unlike that recently reported by Jin et al. (52), we observe in vitro synthetic lethality of ATR and Wee1 inhibition not only in triple-negative breast cancer cells, but in a wide range of breast cancer cell lines, including luminal A and B cells (MCF7, T47-D, MDA-MB-175-VII, and Zr-75-1) and Her2-amplified Sk-Br-3. While Jin et al. speculate that p53 mutation sensitizes cancer cells to combined ATR/Wee1 inhibition, we noticed strong synthetic lethality also in p53wild-type cells, such as MDA-MB-175-VII, Zr-75-1, and MCF7, and the osteosarcoma cell line U-2 OS. Based on our model, in which ATR and Wee1 inhibition leads to decreased S, S/G2, and G2/M checkpoint activation - supported by the recent finding that ATR 
is an important regulator of the S/G2 checkpoint (61) - and subsequently leads to mitotic catastrophe, we speculate that p53 status is less of a predictor of therapeutic outcome by the drug combination than baseline levels of DNA damage and alterations in the mechanisms regulating CDK1/2 activity. Indeed, our unpublished data show that factors besides Chk1 and Wee1 regulating CDK1 activity, such as the Wee1-related kinase Myt1, or factors involved in processing replication-stress intermediates play important roles in cellular sensitivity to Wee1/ATR combination treatment in vitro. Initial clinical trials are expected in cancer types known for their genomic instability, such as cancers with ATM loss, which was previously shown by us to sensitize to ATR inhibition (10), and certain breast, ovarian, or colorectal cancers, where homologous recombination (e.g., by BRCA loss) or other repair pathways are impaired. However, the conditional synthetic lethality underlying combined AZD1775/AZD6738 treatment is based on the increased DNA damage per se in cancer cells compared with normal tissue. This genotoxic stress can have various origins, from aneuploidy to genetic or epigenetic defects, but will result in replication stress. Unfortunately, while several candidate predictive biomarkers have been identified for cellular sensitivity to ATR or Wee1 inhibitors (reviewed in ref. 62), clinical biomarkers for DNA replication stress are still lacking. Whereas in vitro FANCD2 or RAD51 foci resulting from the recruitment of these proteins to common fragile sites are good surrogate markers for replication stress $(63,64)$, attempts to use Ki67, cyclin E, POLD3, $\gamma \mathrm{H} 2 \mathrm{AX}$, and FANCD2 staining in cancer specimens by immunohistochemistry have been disappointing (65). Incidentally, ATR activation should correlate with replication stress and future studies will assess whether ATR phosphorylation at T1989 in cancer biopsies, used as marker of ATR activation in our xenografts, is a predictive biomarker for combined ATR/Wee1-inhibitor treatment.

\section{Methods}

Cell synchronization and cell cycle analysis. U-2 OS cells were treated with $2 \mathrm{mM}$ thymidine for 16 hours, released into fresh medium for 4 hours, followed by nocodazole $(100 \mathrm{ng} / \mathrm{ml})$ treatment for 8 hours. Six hours after release from nocodazole into fresh medium, cells were treated with DMSO, AZD6738 $(1 \mu \mathrm{M})$, AZD1775 $(0.3 \mu \mathrm{M})$, or a combination of AZD6738 and AZD1775.

MDA-MB-231 cells were treated with $2 \mathrm{mM}$ thymidine for 18 hours, followed by release into fresh medium for 8 hours and a second treatment with $2 \mathrm{mM}$ thymidine for 18 hours. After release, cells were treated with DMSO, or AZD6738 $(1 \mu \mathrm{M})$, or AZD1775 $(0.3 \mu \mathrm{M})$, or combined AZD6738 and AZD1775.

For cell cycle analysis, cells were harvested at 2-hour intervals and fixed with $70 \%$ chilled ethanol for at least 24 hours at $-20^{\circ} \mathrm{C}$ before a wash with $1 \times$ PBS. Pelleted cells ( $300 \mathrm{~g}, 5$ minutes) were resuspended in propidium iodide buffer $(50 \mu \mathrm{g} / \mathrm{ml})$ containing RNAse A $(10 \mu \mathrm{g} / \mathrm{ml})$ and incubated at $37^{\circ} \mathrm{C}$ for 30 minutes. Samples were analyzed on a BD FACSCanto II flow cytometer.

Orthotopic breast cancer xenografts and drug treatments. All mice were obtained from breeding colonies at the University of Alberta. For tumor formation, $2 \times 10^{6}$ MDA-MB-231-fluc2-tdT cells were mixed with Matrigel (Corning) and PBS (1:1) and injected in $50 \mu \mathrm{l}$ orthotopically into the inguinal mammary fat pad of 6- to 8-weekold female NOD.Cg-Prkd csid $I l 2 r g^{t m l W j l} / \mathrm{SzJ}$ (NSG) mice. Tumor growth was measured every 4 days using a Vernier caliper and volume was assessed as (length $\times$ width $\left.^{2}\right) / 2$. When the tumor volumes reached approximately $40-50 \mathrm{~mm}^{3}$, mice were randomly segregated into 4 groups ( $n=9$ per group). Mice were treated daily were with vehicle, $25 \mathrm{mg} / \mathrm{kg}$ AZD6738 (in 10\% DMSO, 40\% polypropylene glycol, and $50 \% \mathrm{ddH}_{2} \mathrm{O}$ ), $60 \mathrm{mg} / \mathrm{kg} \mathrm{AZD1775}$ (in 0.5\% methylcellulose), or a combination of AZD6738 and AZD1775 via oral gavage for 26 days. Body weight was measured every 4 days as an indicator of toxicity. Mice were euthanized when the tumor volume reached a total of $1,000 \mathrm{~mm}^{3}$, after a greater than $10 \%$ reduction in body weight, or any other indications of physical discomfort.

For histological studies, tumor-bearing mice $\left(250-\mathrm{mm}^{3}\right.$ tumor volume) were treated with either vehicle or $25 \mathrm{mg} / \mathrm{kg}$ AZD6738 or 60 $\mathrm{mg} / \mathrm{kg}$ AZD1775 or a combination of AZD6738 and AZD1775 via oral gavage for 5 (short term) or 26 (long term) days. Tumors and small intestines (ilea), lungs, livers, kidneys, and spleens were harvested and fixed with $10 \%$ formalin for 48 hours prior to embedding.

Statistics. All statistical analysis was performed using GraphPad Prism 7 software. All experiments were performed at least 3 times with triplicate or quadruplicate samples. $P$ values were calculated using 1-way ANOVA, 2-way ANOVA, and log-rank (Mantel-Cox) test. $P<0.05$ was considered significant, and $P<0.001$ was considered highly significant.

Study approval. All animal studies described were carried out under protocol AC16225 approved by the Cross Cancer Institute's Animal Care Committee, Edmonton, Canada.

Details of materials and other methods, such as survival assays, side population and mammosphere assays, immunofluorescence and immunohistochemistry, microscopy, bioluminescence imaging, and hematopoietic progenitor studies are described in the supplemental material.

\section{Author contributions}

$\mathrm{ABB}, \mathrm{CWL}, \mathrm{GKC}$, and $\mathrm{AMG}$ designed the experiments. $\mathrm{ABB}$, CWL, JJP, and DL performed crystal violet assays. ABB, CWL, and $A M G$ performed in vitro experiments and analyzed the data. $\mathrm{ABB}$ performed in vivo experiments and analyzed the data. $\mathrm{ABB}$ performed statistical analysis. GKC and AMG conceived the data. $\mathrm{ABB}$ and $\mathrm{AMG}$ wrote the manuscript.

\section{Acknowledgments}

We thank Kristi Baker for the C57BL/6 mice; David Murray for helpful suggestions on the manuscript; Powel Crosley for help with R programming; John Lewis for the use of the in vivo imaging system; Xuejun Sun and Geraldine Barron for assistance in the cell imaging facility; Sabina Baghirova and Aja Rieger for assistance with flow cytometry; and AstraZeneca for compounds AZD6738 and AZD1775. ABB and CWL are supported by Alberta Cancer Foundation's Dr. Cyril M. Kay Graduate Scholarship and NSERC Alexander Graham Bell Canada Graduate Scholarship respectively. This research is funded by a startup grant from the University of Alberta and a CIHR grant in the Gamper laboratory, and by NSERC and the Cancer Research Society in the Chan laboratory.

Address correspondence to: Armin Gamper, Department of Oncology. University of Alberta, Cross Cancer Institute, 11560 University Avenue, Edmonton, Alberta T6G 1Z2, Canada. Phone: 780.432.8436; Email: gamper@ualberta.ca. 
1. Ciccia A, Elledge SJ. The DNA damage response: making it safe to play with knives. Mol Cell. 2010;40(2):179-204.

2. Luo J, Solimini NL, Elledge SJ. Principles of cancer therapy: oncogene and non-oncogene addiction. Cell. 2009;136(5):823-837.

3. Drosos Y, et al. ATM-deficiency increases genomic instability and metastatic potential in a mouse model of pancreatic cancer. Sci Rep. 2017;7(1):11144.

4. Bryant HE, et al. Specific killing of BRCA2-deficient tumours with inhibitors of poly(ADP-ribose) polymerase. Nature. 2005;434(7035):913-917.

5. Farmer $\mathrm{H}$, et al. Targeting the DNA repair defect in BRCA mutant cells as a therapeutic strategy. Nature. 2005;434(7035):917-921.

6. O'Neil NJ, Bailey ML, Hieter P. Synthetic lethality and cancer. Nat Rev Genet. 2017;18(10):613-623.

7. Lecona E, Fernández-Capetillo O. Replication stress and cancer: it takes two to tango. Exp Cell Res. 2014;329(1):26-34.

8. Halazonetis TD, Gorgoulis VG, Bartek J. An oncogene-induced DNA damage model for cancer development. Science. 2008;319(5868):1352-1355.

9. Lewis $\mathrm{CW}$, et al. Prolonged mitotic arrest induced by Wee1 inhibition sensitizes breast cancer cells to paclitaxel. Oncotarget. 2017;8(43):73705-73722.

10. Gamper AM, Rofougaran R, Watkins SC, Greenberger JS, Beumer JH, Bakkenist CJ. ATR kinase activation in G1 phase facilitates the repair of ionizing radiation-induced DNA damage. Nucleic Acids Res. 2013;41(22):10334-10344.

11. Kibe T, Zimmermann M, de Lange T. TPP1 blocks an ATR-mediated resection mechanism at telomeres. Mol Cell. 2016;61(2):236-246.

12. Buisson R, et al. Coupling of homologous recombination and the checkpoint by ATR. Mol Cell. 2017;65(2):336-346.

13. Malumbres M, Barbacid M. Mammalian cyclindependent kinases. Trends Biochem Sci. 2005;30(11):630-641.

14. Chow JP, Poon RY, Ma HT. Inhibitory phosphorylation of cyclin-dependent kinase 1 as a compensatory mechanism for mitosis exit. Mol Cell Biol. 2011;31(7):1478-1491.

15. Visconti R, et al. The Fcp1-Wee1-Cdk1 axis affects spindle assembly checkpoint robustness and sensitivity to antimicrotubule cancer drugs. Cell Death Differ. 2015;22(9):1551-1560.

16. Visconti R, Palazzo L, Della Monica R, Grieco D. Fcp1-dependent dephosphorylation is required for M-phase-promoting factor inactivation at mitosis exit. Nat Commun. 2012;3:894.

17. Vassilopoulos A, et al. WEE1 murine deficiency induces hyper-activation of APC/C and results in genomic instability and carcinogenesis. Oncogene. 2015;34(23):3023-3035.

18. Beck $\mathrm{H}$, et al. Regulators of cyclin-dependent kinases are crucial for maintaining genome integrity in S phase. J Cell Biol. 2010;188(5):629-638.

19. Hauge S, et al. Combined inhibition of Wee1 and Chk1 gives synergistic DNA damage in S-phase due to distinct regulation of CDK activity and CDC45 loading. Oncotarget. 2017;8(7):10966-10979.

20. Domínguez-Kelly R, et al. Wee1 controls genomic stability during replication by regulating the Mus81-Eme1 endonuclease. J Cell Biol. 2011;194(4):567-579.
21. Brown EJ, Baltimore D. ATR disruption leads to chromosomal fragmentation and early embryonic lethality. Genes Dev. 2000;14(4):397-402.

22. de Klein A, et al. Targeted disruption of the cell-cycle checkpoint gene ATR leads to early embryonic lethality in mice. Curr Biol. 2000;10(8):479-482.

23. Feoktistova M, Geserick P, Leverkus M. Crystal violet assay for determining viability of cultured cells. Cold Spring Harb Protoc. 2016;2016(4):pdb.prot087379.

24. Foucquier J, Guedj M. Analysis of drug combinations: current methodological landscape. Pharmacol Res Perspect. 2015;3(3):e00149.

25. Técher H, et al. Signaling from Mus81-Eme2dependent DNA damage elicited by Chk1 deficiency modulates replication fork speed and origin usage. Cell Rep. 2016;14(5):1114-1127.

26. Sausville E, et al. Phase I dose-escalation study of AZD7762, a checkpoint kinase inhibitor, in combination with gemcitabine in US patients with advanced solid tumors. Cancer Chemother Pharmacol. 2014;73(3):539-549.

27. Seto T, et al. Phase I, dose-escalation study of AZD7762 alone and in combination with gemcitabine in Japanese patients with advanced solid tumours. Cancer Chemother Pharmacol. 2013;72(3):619-627.

28. Toledo LI, et al. A cell-based screen identifies ATR inhibitors with synthetic lethal properties for cancer-associated mutations. Nat Struct Mol Biol. 2011;18(6):721-727.

29. Reaper PM, et al. Selective killing of ATM- or p53-deficient cancer cells through inhibition of ATR. Nat Chem Biol. 2011;7(7):428-430.

30. O’Driscoll M, Ruiz-Perez VL, Woods CG, Jeggo PA, Goodship JA. A splicing mutation affecting expression of ataxia-telangiectasia and Rad3related protein (ATR) results in Seckel syndrome. Nat Genet. 2003;33(4):497-501.

31. Eriksson D, Stigbrand T. Radiation-induced cell death mechanisms. Tumour Biol. 2010;31(4):363-372.

32. Surova O, Zhivotovsky B. Various modes of cell death induced by DNA damage. Oncogene. 2013;32(33):3789-3797.

33. Gascoigne KE, Taylor SS. Cancer cells display profound intra- and interline variation following prolonged exposure to antimitotic drugs. Cancer Cell. 2008;14(2):111-122.

34. Brinkley BR, et al. Movement and segregation of kinetochores experimentally detached from mammalian chromosomes. Nature. 1988;336(6196):251-254.

35. Beeharry N, Rattner JB, Caviston JP, Yen T. Centromere fragmentation is a common mitotic defect of S and G2 checkpoint override. Cell Cycle. 2013;12(10):1588-1597.

36. Shaner NC, Campbell RE, Steinbach PA, Giepmans $\mathrm{BN}$, Palmer AE, Tsien RY. Improved monomeric red, orange and yellow fluorescent proteins derived from Discosoma sp. red fluorescent protein. Nat Biotechnol. 2004;22(12):1567-1572.

37. Stewart E, et al. Orthotopic patient-derived xenografts of paediatric solid tumours. Nature. 2017;549(7670):96-100.

38. Nam EA, Zhao R, Glick GG, Bansbach CE, Friedman DB, Cortez D. Thr-1989 phosphorylation is a marker of active ataxia telangiectasia-mutated and Rad3-related (ATR) kinase. J Biol Chem. 2011;286(33):28707-28714.

39. Barker N, et al. Identification of stem cells in small intestine and colon by marker gene Lgr5. Nature. 2007;449(7165):1003-1007.

40. Withers HR. Regeneration of intestinal mucosa after irradiation. Cancer. 1971;28(1):75-81.

41. Mohrin M, et al. Hematopoietic stem cell quiescence promotes error-prone DNA repair and mutagenesis. Cell Stem Cell. 2010;7(2):174-185.

42. Akashi K, Traver D, Miyamoto T, Weissman IL. A clonogenic common myeloid progenitor that gives rise to all myeloid lineages. Nature. 2000;404(6774):193-197.

43. Kondo M, Weissman IL, Akashi K. Identification of clonogenic common lymphoid progenitors in mouse bone marrow. Cell.1997;91(5):661-672.

44. Telford WG, Bradford J, Godfrey W, Robey RW, Bates SE. Side population analysis using a violet-excited cell-permeable DNA binding dye. Stem Cells. 2007;25(4):1029-1036.

45. Hanahan D, Weinberg RA. Hallmarks of cancer: the next generation. Cell. 2011;144(5):646-674.

46. Kotsantis P, Petermann E, Boulton SJ. Mechanisms of oncogene-induced replication stress: jigsaw falling into place. Cancer Discov. 2018;8(5):537-555.

47. Greenman C, et al. Patterns of somatic mutation in human cancer genomes. Nature. 2007;446(7132):153-158.

48. Parikh RA, et al. Upregulation of the ATR-CHEK1 pathway in oral squamous cell carcinomas. Genes Chromosomes Cancer. 2014;53(1):25-37.

49. Abdel-Fatah TM, et al. Untangling the ATRCHEK1 network for prognostication, prediction and therapeutic target validation in breast cancer. Mol Oncol. 2015;9(3):569-585.

50. Santamaría D, et al. Cdk1 is sufficient to drive the mammalian cell cycle. Nature. 2007;448(7155):811-815.

51. Matheson CJ, Backos DS, Reigan P. Targeting WEE1 Kinase in Cancer. Trends Pharmacol Sci. 2016;37(10):872-881.

52. Jin J, et al. Combined inhibition of ATR and WEE1 as a novel therapeutic strategy in triple-negative breast cancer. Neoplasia. 2018;20(5):478-488.

53. Toledo LI, et al. ATR prohibits replication catastrophe by preventing global exhaustion of RPA. Cell. 2013;155(5):1088-1103.

54. Kabeche L, Nguyen HD, Buisson R, Zou L. A mitosis-specific and $\mathrm{R}$ loop-driven ATR pathway promotes faithful chromosome segregation. Science. 2018;359(6371):108-114.

55. Forment JV, O'Connor MJ. Targeting the replication stress response in cancer. Pharmacol Ther. 2018;188:155-167.

56. Vitale I, Manic G, De Maria R, Kroemer G, Galluzzi L. DNA damage in stem cells. Mol Cell. 2017;66(3):306-319.

57. Bao $\mathrm{S}$, et al. Glioma stem cells promote radioresistance by preferential activation of the DNA damage response. Nature. 2006;444(7120):756-760.

58. Bartucci M, et al. Therapeutic targeting of Chk1 in NSCLC stem cells during chemotherapy. Cell Death Differ. 2012;19(5):768-778.

59. Gallmeier E, et al. Inhibition of ataxia telangiec- 
tasia- and Rad3-related function abrogates the in vitro and in vivo tumorigenicity of human colon cancer cells through depletion of the CD133(+) tumor-initiating cell fraction. Stem Cells. 2011;29(3):418-429.

60. Toledo CM, et al. Genome-wide CRISPR-Cas9 screens reveal loss of redundancy between PKMYT1 and WEE1 in glioblastoma stem-like cells. Cell Rep. 2015;13(11):2425-2439.
61. Saldivar JC, et al. An intrinsic S/G2 checkpoint enforced by ATR. Science. 2018;361(6404):806-810.

62. Brandsma I, Fleuren EDG, Williamson CT, Lord CJ. Directing the use of DDR kinase inhibitors in cancer treatment. Expert Opin Investig Drugs. 2017;26(12):1341-1355.

63. Chan KL, Palmai-Pallag T, Ying S, Hickson ID. Replication stress induces sister-chromatid bridging at fragile site loci in mitosis. Nat Cell Biol. 2009;11(6):753-760.

64. Schwartz M, et al. Homologous recombination and nonhomologous end-joining repair pathways regulate fragile site stability. Genes Dev. 2005;19(22):2715-2726.

65. Ren L, et al. Potential biomarkers of DNA replication stress in cancer. Oncotarget. 2017;8(23):36996-37008. 